\title{
Numerical investigation of transitional supersonic axisymmetric wakes
}

\author{
By RICHARD D. SANDBERG AND HERMANN F. FASEL \\ Department of Aerospace and Mechanical Engineering, University of Arizona, Tucson, AZ 85721, USA
}

(Received 8 July 2005 and in revised form 23 January 2006)

Transitional supersonic axisymmetric wakes are investigated by conducting various numerical experiments. The main objective is to identify hydrodynamic instability mechanisms in the flow at $M=2.46$ for several Reynolds numbers, and to relate these to coherent structures that are found from various visualization techniques. The premise for this approach is the assumption that flow instabilities lead to the formation of coherent structures. Three high-order accurate compressible codes were developed in cylindrical coordinates for this work: a spatial Navier-Stokes (N-S) code to conduct direct numerical simulations (DNS), a linearized N-S code for linear stability investigations using axisymmetric basic states, and a temporal N-S code for performing local stability analyses. The ability of numerical simulations to exclude physical effects deliberately is exploited. This includes intentionally eliminating certain azimuthal/helical modes by employing DNS for various circumferential domain sizes. With this approach, the impact of structures associated with certain modes on the global wake-behaviour can be scrutinized. Complementary spatial and temporal calculations are carried out to investigate whether instabilities are of local or global nature. Circumstantial evidence is presented that absolutely unstable global modes within the recirculation region co-exist with convectively unstable shear-layer modes. The flow is found to be absolutely unstable with respect to modes $k>0$ for $R e_{D}>5000$ and with respect to the axisymmetric mode $k=0$ for $R e_{D}>100000$. It is concluded that azimuthal modes $k=2$ and $k=4$ are the dominant modes in the trailing wake, producing a 'four-lobe' wake pattern. Two possible mechanisms responsible for the generation of longitudinal structures within the recirculation region are suggested.

\section{Introduction}

It is well known that for subsonic (incompressible) wakes, the dynamics of large (coherent) structures play a dominant role in the local and global behaviour of the flow. This evidence was found from both experimental investigations and numerical simulations and was confirmed by theoretical studies. For example, in experiments of the flow around a sphere, Aschenbach (1974) detected large structures and identified two different modes of vortex shedding: a shear-layer mode for moderate Reynolds numbers with a Strouhal number increasing with Reynolds number, and a second mode of shedding that has an approximately constant Strouhal number. Aschenbach showed that the shedding from the second mode was in the form of two counterrotating helices. Monkewitz (1988) found analytically that the first helical mode is absolutely unstable in the near wake behind a bluff body for Reynolds numbers in excess of 3300 (based on wake-diameter and free-stream velocity). Monkewitz also found that large-scale structures co-exist with a recirculation region, which was later 
confirmed experimentally by Cannon \& Champagne (1991). The experiments also revealed that these large-scale structures are predominantly helical with a dominant first azimuthal mode. Using numerical simulations in conjunction with linear stability theory (LST), absolute/global instabilities were found for a two-dimensional bluff body with a blunt base by Hannemann \& Oertel (1989). For three-dimensional results, see Oertel \& Delfs (1995). In addition, numerical simulations for an axisymmetric body with a blunt base (Schwarz, Bestek \& Fasel 1994) revealed the existence of absolutely/globally unstable modes that are of helical nature.

For supersonic flows, on the other hand, little is known yet about the origin and dynamical behaviour of large coherent structures in the near-wake region behind an axisymmetric body. This certainly cannot be attributed to a lack of interest in the physical problem. On the contrary, a detailed understanding of the physical mechanisms in the near-wake region could be highly rewarding in terms of reducing drag of flight vehicles at supersonic speeds (Rollstin 1990, showed that the base drag, caused by the low mean pressure at the base, accounts for up to $35 \%$ of the total drag). The scarcity of knowledge concerning this particular flow scenario originates rather in the complexity of the physical problem and difficulties, uncertainties and high costs associated with experiments. For instance, wind-tunnel experiments suffered from the difficulty of properly supporting the (axisymmetric) base model so as not to cause undue effects on the flow field behind the base (cf. Chapman 1951; Donaldson 1955; Dayman 1963). Other factors that can compromise experimentally obtained data include the use of intrusive probes and wind-tunnel interference, i.e. vibrations, upstream sound, noise radiated from turbulent boundary layers of the wind-tunnel walls and blockage, to name a few. The strong sensitivity of the flow to perturbations (in the form of sound, solid surfaces, such as probes, etc.) is most probably due to a global instability that may be present in the recirculation region. Therefore, most of the early work (cf. Demetriades 1968a, $b$ ) employing intrusive probes was restricted to the far wake. Nevertheless, some quantitative evidence of the existence of dominant large structures in supersonic axisymmetric wake flows was provided; amplitude spectra (see figures 11 to 14 in Demetriades 1968b) displayed distinct peaks at relatively low frequencies, thus indicating the presence of dominant modes (structures). In addition, the amplitude distribution for the velocity fluctuations (figures 2 and 3, respectively, of Demetriades 1968b) resembled that of an incompressible axisymmetric wake, where it is known that the resulting profile is due to the presence of dominant large coherent structures (see, e.g. Cannon \& Champagne 1991). For supersonic jet flows, Crow \& Champagne (1971) observed coherent structures in their experiments (calling them 'orderly structures'). Large coherent structures were also observed in experiments of supersonic jets by Oertel (1979).

The use of a forward sting support for experiments of supersonic axisymmetric wakes in a specifically constructed supersonic blowdown-type wind tunnel (see, e.g. Herrin \& Dutton 1994, 1995) appears to have alleviated many of the above-mentioned problems of experimental investigations. In addition, the use of the non-intrusive laserDoppler velocimetry (LDV) technique made the investigation of the near-wake region possible. Bourdon \& Dutton (1999) were able to show clearly the presence of large coherent structures in the axisymmetric base flow by employing planar visualizations. The observed structures appeared to be of significant size relative to the shear-layer thickness. Thus, it appears safe to say that large coherent structures are present in supersonic axisymmetric flows and may indeed play an important role.

There is considerable evidence that the formation of large-scale structures is due to hydrodynamic instabilities of the (time-averaged) mean flow and that the 
development of these structures can be captured by stability theory (see, e.g. Gaster, Kit \& Wygnanski 1985; Oertel 1990; Theofilis 2003). In fact, certain aspects of the development can be captured with linear stability theory, although intensities (amplitudes) of the structures are often too large for the linear stability theory to be valid. Experimental results for incompressible turbulent mixing layers, twodimensional turbulent wakes, and axisymmetric wakes with a blunt base have shown that certain key features, such as dominant frequencies, mode shapes (amplitude distributions), and streamwise spacing (streamwise wavelengths) of the structures can be predicted well by linear stability theory (Wygnanski, Champagne \& Marasli 1986; Marasli, Champagne \& Wygnanski 1989). These investigations support the notion that hydrodynamic instabilities give rise to the generation of large-scale structures and affect their spatial and temporal development.

For the present investigation, numerical experiments were conducted exploiting the ability of numerical simulations to exclude physical effects deliberately and, therefore assess their importance. Three separate approaches were employed: direct numerical simulations (DNSs); temporal DNS (TDNS); and linearized Navier-Stokes (LNS) calculations. The motivation for each of the methods is given in the following.

Classical linear stability theory (LST) deals with instabilities of infinitesimally small amplitudes that develop in a parallel flow, containing only one inhomogeneous spatial direction. Mathematically, the problem can be formulated as an eigenvalue problem (EVP) that requires the solution of the system of the Orr-Sommerfeld and Squire equations. The coupling of slightly damped solutions of the EVP can lead to an algebraic, or transient, growth; however, in the current investigation, only the classical view of unstable modes leading to transition will be considered. In spite of (or because of) the restrictions of parallel flow theory, LST has been applied by many workers. For example, Huerre \& Monkewitz (1985) presented the distinction between convective and absolute instabilities in parallel shear flows, a terminology that originated from plasma physics. According to their definition, a flow is convectively unstable if a pulse disturbance decays to zero in time at all locations in the flow. On the other hand, an absolute instability is present if a disturbance leads to exponential growth in the entire field. Here, instead of solving the EVP, local stability investigations of a parallel basic state are conducted employing a temporal compressible Navier-Stokes code in cylindrical coordinates (for a detailed discussion of the spatial versus temporal approach see Fasel 1990). This method was chosen because it also allowed for local stability calculations of disturbances with nonlinear amplitudes. It is sought to exploit the fact that the effects caused by streamline curvature, pressure gradients, shocks and expansion waves, etc. are eliminated by this model. In the present investigation, by comparing with results from spatial calculations, the importance of the omitted physical mechanisms can be evaluated.

The mean flow topology of the near-wake region downstream of an axisymmetric cylinder with a blunt base is shown schematically in figure 1 . The supersonic approach boundary layer (i) separates at the body corner producing an expansion (ii) with a large turning angle. A thin, yet very energetic free shear-layer forms (iii) separating the outer inviscid fluid from a large recirculation region (iv) downstream of the base. A recompression (v) process realigns the flow with the axis of symmetry and subjects the shear layer to a strong adverse pressure gradient. Downstream of the mean reattachment point (vi) a trailing wake (vii) develops. It is evident that the mean basic state is strongly non-homogeneous in the streamwise direction. Thus, it is desirable to conduct stability investigations accounting for the strong non-parallel effects of the basic state. 


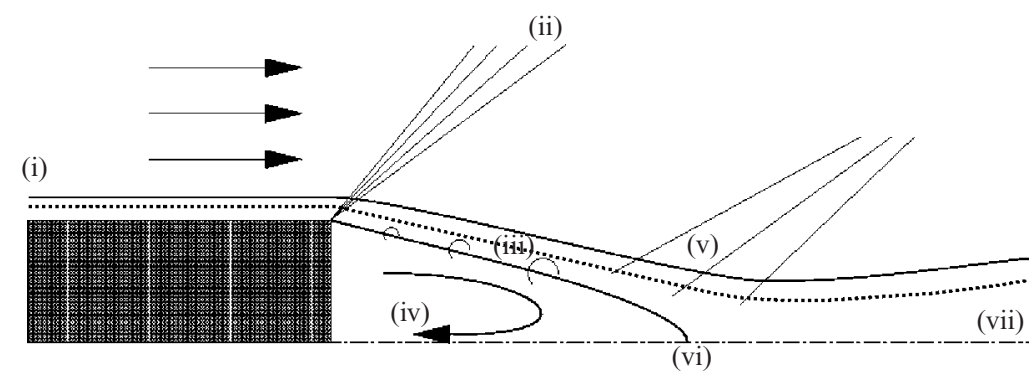

FIGURE 1. Schematic of the mean flow field of a supersonic base flow; dotted line denotes the sonic line.

In an extensive review on different classes of instabilities for spatially growing flows, Huerre \& Monkewitz (1990) introduced the concept of global instability in the context of slow variations of the basic flow in the streamwise direction. Another approach for solving the stability equations where the basic state features a weak dependence of the streamwise coordinate was accomplished by the derivation of the parabolized stability equations (Herbert 1997). If, however, the basic state is truly nonparallel, another approach is required. A multidimensional eigenvalue problem can be solved with the underlying basic state being a fully two-dimensional steady or timeperiodic non-parallel flow, as presented in Theofilis (2003). The numerical integration of the LNS equations appears to be an efficient alternative for conducting linear stability investigations of a strongly non-parallel, steady or time-periodic basic state. Therefore, an LNS code in cylindrical coordinates was developed for linear stability investigations using a two-dimensional basic state. In particular, by conducting spatial and temporal simulations in a complementary fashion, instabilities could be identified as local or global modes. Using the temporal approach, it can be determined whether disturbances are locally unstable in the absence of the global effects mentioned above.

Once the nonlinear behaviour becomes of interest, which is the case when investigating disturbances with nonlinear amplitudes (such as coherent structures), the full Navier-Stokes equations must be solved, i.e. DNSs must be conducted. Thus, a high-order accurate compressible Navier-Stokes (N-S) solver in cylindrical coordinates was developed. As mentioned above, the flow contains a large variety of difficulties such as the combination of shock waves, thin free shear layers, boundary layers, and recirculating regions that have to be captured correctly in a numerical simulation. In addition, high-Reynolds-number flows exhibit a very broad spectrum of time and length scales that, in the case of DNSs, must be fully resolved by the computational grid. For that reason, fully resolved DNSs of wakes at Reynolds numbers of the order of $O\left(10^{6}\right)$, as investigated in experiments by Dutton and coworkers, are out of reach, even with present supercomputers, and probably will be for a considerable time. Therefore, transitional supersonic base flows were chosen for stability investigations using DNS. Although at the lower Reynolds numbers investigated here, the flow is not fully turbulent, it was shown that many of the qualitative features found in the experiments by Dutton and co-workers can be captured (see, e.g. Fasel, Von Terzi \& Sandberg 2006; Sandberg \& Fasel 2004, 2006). Moreover, transitional flows facilitate the investigation of the initial development of the large-scale structures because the breakdown to small-scale turbulence either does not fully occur, or takes place on a relatively slow time scale, leading to a well-defined separation of scales. 
The objective of this work is to contribute towards the understanding of the origin and the dynamics of coherent structures in supersonic axisymmetric wakes behind bluff bodies. The premise for this study is the underlying assumption that flow instabilities lead to the formation of coherent structures and determine their evolution. In $\S \S 2$ and 3 , the governing equations and the numerical methods of the numerical codes are presented, respectively. Section 4 first presents the results obtained from linear stability investigations, subdivided into results from spatial and temporal simulations. Then DNS results are discussed with an emphasis on coherent structures followed by mean flow data. All results are summarized in $\S 5$ and numerical details are given in the Appendix.

\section{Governing equations}

\subsection{Navier-Stokes equations}

The physical problem under consideration is governed by the full compressible NavierStokes equations, consisting of conservation of mass, momentum and total energy. The flow is assumed to be an ideal gas with constant specific heat coefficients. For simplicity, all equations in this section are presented in tensor notation. For the full set of governing equations in cylindrical coordinates, see Appendix A.

All dimensional quantities, denoted by an asterisk, are made dimensionless using the flow quantities at a reference location in the flow; here the free-stream/inflow location is used. The radius of the body was chosen as the reference length. The nondimensionalization results in the following dimensionless parameters $R e=\rho_{\infty}^{*} u_{\infty}^{*} r^{*} / \mu_{\infty}^{*}$, $M=u_{\infty}^{*} / a_{\infty}^{*}, \operatorname{Pr}=\mu_{\infty}^{*} c_{p}^{*} / \kappa_{\infty}^{*}$ and $E c=u_{\infty}^{* 2} / c_{p}^{*} T_{\infty}^{*}$. The non-dimensional continuity, momentum and the energy equations are:

$$
\begin{gathered}
\frac{\partial \rho}{\partial t}+\frac{\partial}{\partial x_{k}}\left(\rho u_{k}\right)=0, \\
\frac{\partial}{\partial t}\left(\rho u_{i}\right)+\frac{\partial}{\partial x_{k}}\left(\rho u_{i} u_{k}+p \delta_{i k}-\tau_{i k}\right)=0, \\
\frac{\partial}{\partial t}(\rho E)+\frac{\partial}{\partial x_{k}}\left(\rho u_{k} H+q_{k}-u_{i} \tau_{i k}\right)=0,
\end{gathered}
$$

with the total enthalpy $H=E+p / \rho$ where the total energy is defined as $E=c_{v} T+$ $(1 / 2) u_{i} u_{i}$. The molecular-stress tensor and the heat-flux vector are computed as

$$
\tau_{i k}=\frac{\mu}{\operatorname{Re}}\left(\frac{\partial u_{i}}{\partial x_{k}}+\frac{\partial u_{k}}{\partial x_{i}}-\frac{2}{3} \frac{\partial u_{j}}{\partial x_{j}} \delta_{i k}\right), \quad q_{k}=-\frac{\mu}{\operatorname{PrEcRe}} \frac{\partial T}{\partial x_{k}},
$$

respectively, where the Prandtl number is assumed to be constant at $\operatorname{Pr}=0.70$ and the Eckert number is $E c=(\gamma-1) M^{2}$ with $\gamma=1.4$. The pressure is obtained from the equation of state $p=\rho T / \gamma M^{2}$. To close the system of equations, the molecular viscosity $\mu$ and the thermal conductivity are computed according to Sutherland's law (cf. White 1991)

$$
\mu(T)=\kappa(T)=T^{3 / 2}\left(\frac{1+\mathscr{R}_{S u}}{T+\mathscr{R}_{S u}}\right) \quad \text { with } \quad \mathscr{R}_{S u}=\frac{110.6 K}{T_{\infty}^{*}} .
$$

\subsection{Temporal Navier-Stokes simulations}

For temporal simulations, the full Navier-Stokes equations are solved. However, with the radial velocity component of the basic state set to zero and the lack of streamwise gradients of the basic state, the governing equations of the streamwise and azimuthal 
mean flow reduce to a pure diffusion equation. A forcing term must be added to the right-hand side of the governing equations in order to avoid a significant change of the basic state over time. For a detailed discussion of applying forcing terms in order to maintain the basic state, see e.g. Laurien \& Kleiser (1989). In addition, the assumption of a parallel basic state leads to different inflow and outflow boundary conditions as discussed in $\S 3$.

\subsection{Linearized Navier-Stokes equations}

The full compressible Navier-Stokes equations in cylindrical coordinates are used as the basis for obtaining the linearized Navier-Stokes equations. All variables are decomposed into a steady two-dimensional basic-state component and disturbance quantities $\phi=\breve{\phi}+\dot{\phi}$. Terms that contain only basic-state variables can be subtracted because they satisfy the Navier-Stokes equations for the basic state. All products containing more than one disturbance variable are neglected as they are considered small compared to products of disturbance variables and basic state variables. Because only two-dimensional basic states will be considered for the present work $(\breve{w}=0)$, all azimuthal derivatives of basic-state terms vanish. The set of equations that must be solved is derived in detail in Appendix B.

\section{Numerical method}

The governing equations are solved numerically at discrete points. High commonality between the spatial codes and the temporal code was desired in order to allow attributing discrepancies in the solution to physical differences and not to numerical issues. The linearized code is based on the full Navier-Stokes code. The same numerical method was used, keeping close to all subroutines unchanged. Only the transport equations were substituted with the linearized equations.

\subsection{Temporal and spatial discretization}

All codes developed share the same explicit time advancement, employing a standard four-stage Runge-Kutta scheme. A further commonality of all codes is the use of sixth-order accurate split compact finite differences in the radial direction. As described in Sandberg (2004), compact finite-difference stencils are derived that are valid on a non-equidistant grid in contrast to mapping the computational domain to a uniform grid. This method was chosen because it was shown to lead to a lower truncation error (see Meitz \& Fasel 2000). For the spatial calculations, fourthorder accurate standard split differences are used to facilitate a streamwise domaindecomposition. The fourth-order accurate standard split differences are also derived for non-equidistant grids. For temporal simulations, the parallel flow assumption allows for a pseudospectral approach in the streamwise direction, i.e. azimuthal derivatives are taken and the governing equations are advanced in Fourier space; however, nonlinear terms are computed in physical space. Grid stretching for the radial and the streamwise directions was employed to enable clustering of points in regions of high gradients, e.g. in the approach flow boundary layer, the shear layer, and the corner of the after-body. For the current investigation, the grids were generated using polynomial functions. Chung \& Tucker (2003) showed that the accuracy of high-order finite-difference schemes suffers significantly once the grid is very strongly stretched. Therefore, in the radial direction the grid was designed to be equidistant behind the base $(0 \leqslant r \leqslant 1)$ to achieve the highest possible accuracy. By the same token, a large number of grid-points was used in the streamwise direction to enforce 
(a)

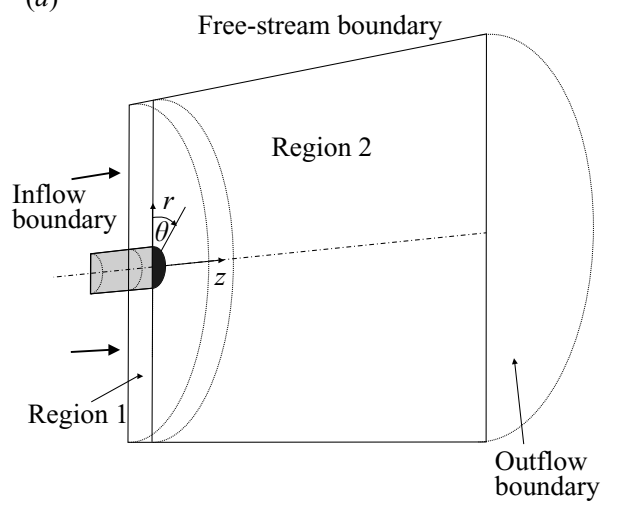

(b)

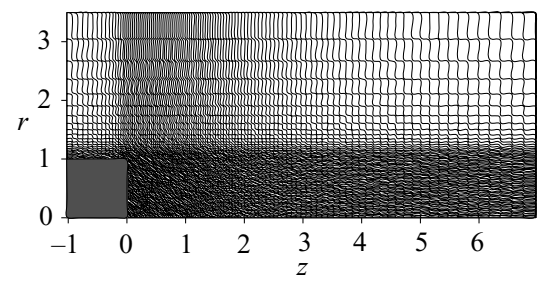

Figure 2. (a) Computational domain and (b) typical streamwise/radial grid for spatial calculations; for clarity, only every fourth grid point is shown in the streamwise and the radial directions in the grid picture.

very benign grid stretching with ratios of $\Delta z_{\max } / \Delta z_{\min } \leqslant 20$. A typical distribution of grid points is shown in figure 2 .

The axisymmetric wake is a natural application for the use of a spectral discretization in the azimuthal direction owing to the periodicity of the physical problem. For a plane geometry, on the other hand, domain-width studies must be conducted to determine which spanwise wavelengths are most amplified because, using Fourier transforms, only integer multiples of the fundamental wavenumber can be captured. For example, when using a domain width of $L_{z}=3$, not only waves with wavelengths larger than $\lambda_{z}>3$ are omitted, but also a disturbance with a wavelength of $\lambda_{z}=2$ would be excluded. In the cylindrical coordinate system, theses studies are not necessary because the geometry requires the wavelength of the disturbance wave to be an integer fraction of the fundamental wave $\left(\lambda_{\theta}=2 \pi\right)$. Thus, the full DNS code and the temporal code employ a pseudospectral approach in the azimuthal direction. Owing to the use of symmetric Fourier transforms, the magnitudes and distributions of azimuthal Fourier modes $k$ and $-k$ will be identical. Therefore, throughout the remainder of this paper, we will not distinguish between positive and negative modes. Note that for simplicity, the technically correct notation of $k= \pm 1, \pm 2, \ldots$ is replaced by $k=1,2, \ldots$. For the linearized code, in the absence of nonlinear terms, a genuine spectral approach can be chosen, advancing the linearized equations entirely in Fourier space. Further details on the spatial and temporal discretization, in particular on the benefits of employing splitting for the finite-difference stencils, are given in Sandberg (2004).

\subsection{Boundary and initial conditions}

The computational domain for a spatial calculation is shown in figure 2. Only the last part of the approach flow is computed (region 1). For the DNS code, the boundary conditions were as follows. At the inflow boundary, the axial and azimuthal velocity and the temperature were set to a constant value. The pressure was fixed in the supersonic region and a Neumann condition was used in the subsonic part of the approach boundary layer. The outflow boundary was computed via onesided stencils, setting the second derivative to zero. The free-stream boundary was represented by Neumann conditions on all variables. Alternatively, a decay condition was implemented requiring the specification of an appropriate wavenumber. At the 

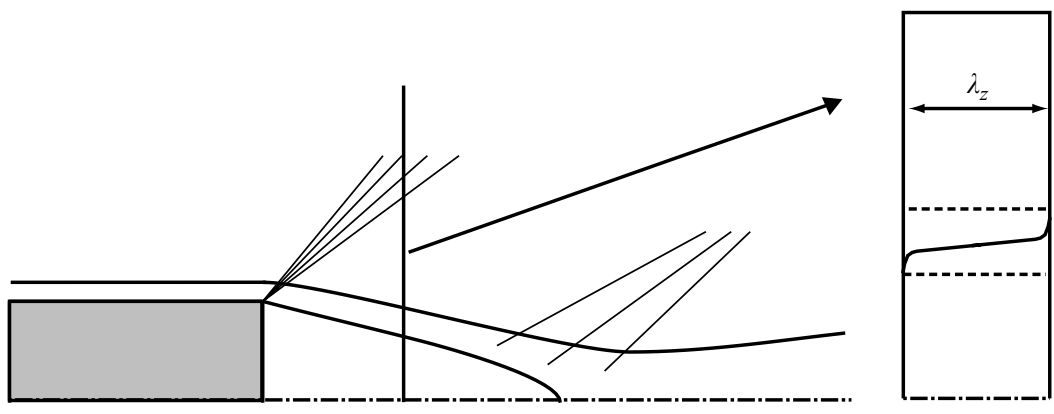

Figure 3. Computational domain for temporal calculations.

walls, no-slip and no-penetration were enforced for all velocity components. The pressure at the wall was computed from the applicable momentum equation, i.e. the radial momentum at the approach flow wall, and the axial momentum for the base wall. An adiabatic wall was enforced by computing temperature through a highorder accurate extrapolation. For all boundaries mentioned above, the density was computed through the equation of state for a perfect gas. For axisymmetric wake calculations, a compressible similarity solution was interpolated onto the grid used in the approach boundary-layer region and extended downstream of the base in the region $1 \leqslant r \leqslant r_{\max }$. Behind the base $(0 \leqslant r<1)$, all variables were set to the value of the corner-point. For three-dimensional calculations, a converged axisymmetric flow field was used as initial condition for the zeroth Fourier mode and the higher modes were set to zero.

In LNS calculations, only the disturbance quantities are solved for, requiring several modifications to the boundary conditions. At walls, in addition to setting the fluctuations of the velocity components to zero, temperature fluctuations were also prohibited, even when an adiabatic boundary condition was used for the basic state. Further, all fluctuations were set to zero at the inflow. All other boundary conditions were treated as in the full N-S code. The initial condition for the linearized calculations always consisted of a two-dimensional basic state that was a converged axisymmetric solution from the full Navier-Stokes code.

For temporal simulations, the streamwise extent of the domain is small to justify the assumption of locally parallel flow. The measure for the domain length thereby is the wavelength of the fundamental instability wave. Hence, periodic boundary conditions can be employed for the inflow and outflow boundaries. All other boundaries were treated as in the spatial DNS. Figure 3 shows the computational domain for temporal wake calculations. The radial profiles of the conservative variables at a specific location were specified as initial conditions for all temporal simulations and constituted the basic state, i.e. the mode $(l, k)=(0,0)$, where $l$ denotes the streamwise Fourier mode. The radial velocity component was set to zero in order to be consistent with the temporal assumption of parallel flow.

\subsection{Axis treatment}

Owing to a pseudospectral approach in the azimuthal direction, the flow field is represented in Fourier modes. A state-of-the-art axis treatment is implemented, exploiting parity conditions. Even parity implies that the even azimuthal modes of a variable have an even symmetry and, conversely, the odd modes have an odd symmetry at the axis. On the other hand, for odd parity, the even azimuthal modes of the variable have an odd symmetry and the odd modes have an even symmetry at 
Variable/equation $\frac{1}{2}$ $\frac{1}{4}$ $\frac{1}{6}$ $\frac{1}{8}$ $\frac{1}{16}$

$\rho, \rho u, \rho E / r$-momentum, Even/odd Even/even Even/odd Even/even Even/even $\theta$-momentum

$\rho v, \rho w /$ continuity, $z$-momentum, energy

Odd/even Odd/odd Odd/even Odd/odd Odd/odd

TABLE 1. Symmetries of computational azimuthal Fourier modes (even modes/odd modes) at the axis for all variables and equations for different azimuthal domain-sizes.

\begin{tabular}{lclllll}
\hline Variable & Mode $k_{N}$ & $\frac{1}{2}$ & $\frac{1}{4}$ & $\frac{1}{6}$ & $\frac{1}{8}$ & $\frac{1}{16}$ \\
$\rho, \rho u, \rho E$ & 0 & Non-zero & Non-zero & Non-zero & Non-zero & Non-zero \\
& 1 & Zero & Zero & Zero & Zero & Zero \\
& 1 & Zero & Zero & Zero & Zero & Zero \\
$\rho v, \rho w$ & 0 & Zero & Zero & Zero & Zero & Zero \\
& 1 & Non-zero & Zero & Zero & Zero & Zero \\
& $>1$ & Zero & Zero & Zero & Zero & Zero
\end{tabular}

TABLE 2. Boundary conditions of computational azimuthal Fourier modes at the axis for all variables for different circumferential domain-sizes.

the axis. A rigorous derivation of the parity can be found in Lewis \& Bellan (1990). The resulting boundary conditions at the axis are as follows. The zeroth azimuthal mode of all quantities evolves according to the governing equations, except for the radial and the azimuthal velocity components, which are set to zero. The first mode of the radial and azimuthal velocity components are permitted to be non-zero at the axis; however, all other higher modes are set to zero at the axis.

A modification of this axis treatment was implemented to allow for calculations of domain sizes with an azimuthal extent of less than $\pi$. In the case of a quartercylinder, the numerical modes $k_{N}=1,2,3, \ldots, k_{\max }$ correspond to the physical modes $k=2,4,6, \ldots, k_{\max }$ in the half-cylinder case, hence all higher modes have to be set to zero at the axis. Also, for the quarter-cylinder case, the parity conditions have to be changed, because all corresponding physical modes are even. For the option of computing $1 / 8$ or $1 / 16$ of a cylinder, only the azimuthal modes $k=4,8,12, \ldots, k_{\max }$ or $k=8,16,24, \ldots, k_{\max }$ are computed, respectively. They require the same axis treatment as in the quarter-cylinder case.

An investigation of $1 / 6$ of a cylinder contains only the physical modes $k=3,6,9, \ldots, k_{\max }$ and therefore, as above, all higher modes are set to zero for this case. However, as opposed to the quarter-, 1/8- or 1/16-cylinder cases, the odd numerical azimuthal modes correspond to odd physical modes (e.g. $k_{N}=1 \rightarrow k=3$ ) and the even numerical azimuthal modes correspond to even physical modes (e.g. $k_{N}=2 \rightarrow k=6$ ). Hence, the same parity conditions as in the half-cylinder case can be used. A compilation of all symmetry and boundary conditions at the axis is given in tables 1 and 2.

\section{Results}

\subsection{Linear stability simulations}

Linear stability calculations of supersonic axisymmetric wakes were carried out at Reynolds numbers between 5000 and 200 000. For all cases, the Mach number was 


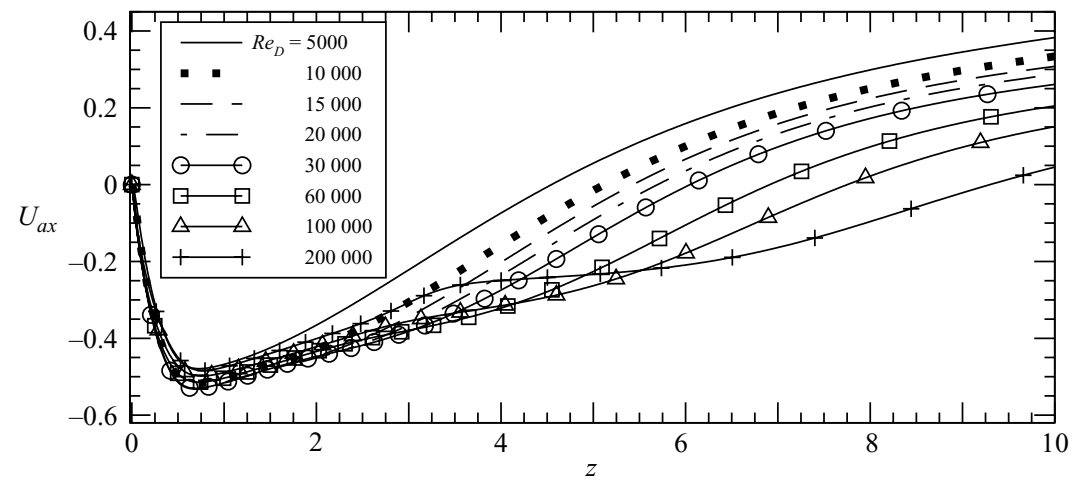

FIGURE 4. Centreline streamwise velocity obtained from axisymmetric N-S calculations for different Reynolds numbers; $\delta_{c}=0.1, M=2.46$.

chosen to be $M=2.46$ to match the flow parameters of experiments by Bourdon \& Dutton (1999) and kept constant to show whether certain trends could be established as a function of Reynolds number only. Both spatial and temporal stability calculations were conducted in order to determine whether local and/or global instabilities are present in the flow. To investigate whether the flows are absolutely unstable with respect to azimuthal/helical perturbations, initial pulse disturbances were introduced within the recirculation region. The amplitudes of the Fourier modes at the disturbance location were monitored over time to determine whether the disturbances grow or decay. For cases where the pulse response decayed in time, periodic forcing was applied to the shear layer and the spatial growth or decay of the perturbations was observed to identify whether the flow is subject to convective instabilities.

\subsubsection{Basic state}

In order to obtain a basic state for the linear stability calculations, axisymmetric calculations were conducted with the full N-S code for Reynolds numbers up to $R e_{D}=200000$. The converged axisymmetric flow fields served as the basic state for the three-dimensional spatial and temporal stability calculations. The boundarylayer thickness at separation, $\delta_{c}$, has a pronounced effect on the global flowfield, even for axisymmetric calculations. To match the approach boundary-layer thickness measured in experiments by Bourdon \& Dutton (1999), the approach boundary layer was designed such that $\delta_{c}=0.1$ for all cases. For all calculations with $R e_{D}<100000$, the computational domain was discretized using $780 \times 130$ points in the streamwise direction and the radial direction, respectively. For larger Reynolds numbers, $1280 \times 160$ points were used. For all cases, the distance from the base to the outflow boundary and from the axis to the free-stream boundary was $z_{\text {out }}-z_{\text {in }}=16$ and $r_{\text {max }}=6$, respectively. The finest grid-spacing was enforced at the base-corner, where $\Delta z_{c}=\Delta r_{c}=0.008$ and the computational time step was $\mathrm{d} t=2.4 \times 10^{-3}$. Several calculations were repeated on different computational grids to verify that the gridresolution was sufficient. The axisymmetric calculations converged to a steady-state solution for all Reynolds numbers $R e_{D} \leqslant 100000$, implying that no absolute instability with respect to axisymmetric disturbances is present. However, for $R e_{D}>100000$, the flow became unsteady, suggesting that the flow may be absolutely unstable with respect to the axisymmetric mode.

The streamwise velocity component at the axis obtained from the axisymmetric $\mathrm{N}-\mathrm{S}$ calculations is shown in figure 4. Similar to incompressible results obtained 
by Schwarz et al. (1994), the maximum reverse velocity is fairly constant for all Reynolds numbers investigated when keeping the approach boundary-layer thickness constant. However, the recirculation length varies considerably and increases for larger Reynolds numbers. The streamwise axis-velocity at the outflow boundary is supersonic for $R e_{D}<20000$. For larger Reynolds number, the flow becomes marginally subsonic close to the axis. Because the cases at $R e_{D}>100000$ became unsteady, an averaged solution is shown.

\subsubsection{Spatial results}

The same computational grids were used for the linear stability simulations as for converging the basic state with the DNS code. Initial pulse disturbances were introduced using the following procedure: higher Fourier modes $(k>0)$ initialized to zero were added to the basic state $(k=0)$. Within the recirculation region, a top-hat function with an extent of six grid points in the streamwise and the radial directions was specified in all Fourier modes of density with a small amplitude (typically of order $10^{-4}$ ). This distribution served as initial condition and, thus, constituted a pulse disturbance in both space and time. For all Reynolds numbers investigated, nine Fourier modes were used. In contrast to temporal calculations employing a Fourier decomposition in the streamwise direction, for each azimuthal mode the most amplified streamwise wavelengths are included in the calculations and cannot be excluded by an unfortunate choice of the streamwise domain length. Note that in the spatial case, the temporal behaviour of each azimuthal mode can be a superposition of multiple unstable streamwise wavelengths. The temporal development of the azimuthal Fourier modes of the streamwise velocity component was monitored at the disturbance point (here $z=2.5$ and $r=0.5$, which is representative of various pulse locations within the recirculation region) and is shown in figure 5 for several Reynolds numbers.

At $R e_{D}=5000$, all azimuthal modes decay rapidly, implying that, at this Reynolds number, the supersonic flow is linearly stable with respect to axisymmetric and helical modes. This illustrates the strong damping effect of compressibility, considering that incompressible wakes are subject to absolute instabilities with respect to helical modes at lower Reynolds numbers (see, e.g. Monkewitz 1988; Hannemann \& Oertel 1989; Schwarz et al. 1994). Even though $k=0$ and $k=1$ are the least damped modes at $R e_{D}=5000$, when increasing the Reynolds number to 10000 , these two modes still are not amplified, but the azimuthal Fourier modes $k=2$ and $k=3$ become amplified. It should be noted that mode $k=2$ grows monotonically, while mode $k=3$ and several higher modes exhibit an oscillatory behaviour in time with a regular period of $F \approx 12$. The absolute values of the data are shown in order to display the results on a logarithmic scale; therefore, sign changes appear as local minima. Further increasing the Reynolds number to $R e_{D}=30000$, the first azimuthal mode also becomes unstable and modes $k=1-4$ all exhibit monotone growth with modes $k=3$ and $k=4$ featuring the largest growth rates. At this point, it would be of interest to solve the multidimensional eigenvalue problem for the basic states under consideration according to Theofilis (2003). This could determine whether the modes exhibiting monotonous growth possess a purely imaginary complex frequency, i.e. are non-oscillatory.

The main difference that can be observed for both the $R e_{D}=60000$ and $R e_{D}=100000$ cases (besides the increased amplification rates) is that modes $k=7$ and $k=8$ possess growth rates exceeding those of most other modes, except for $k=3$ and $k=4$. Only when increasing the Reynolds number to 200000 , can a qualitative change be noticed. For this case, mode $k=1$ is the most amplified Fourier mode and 

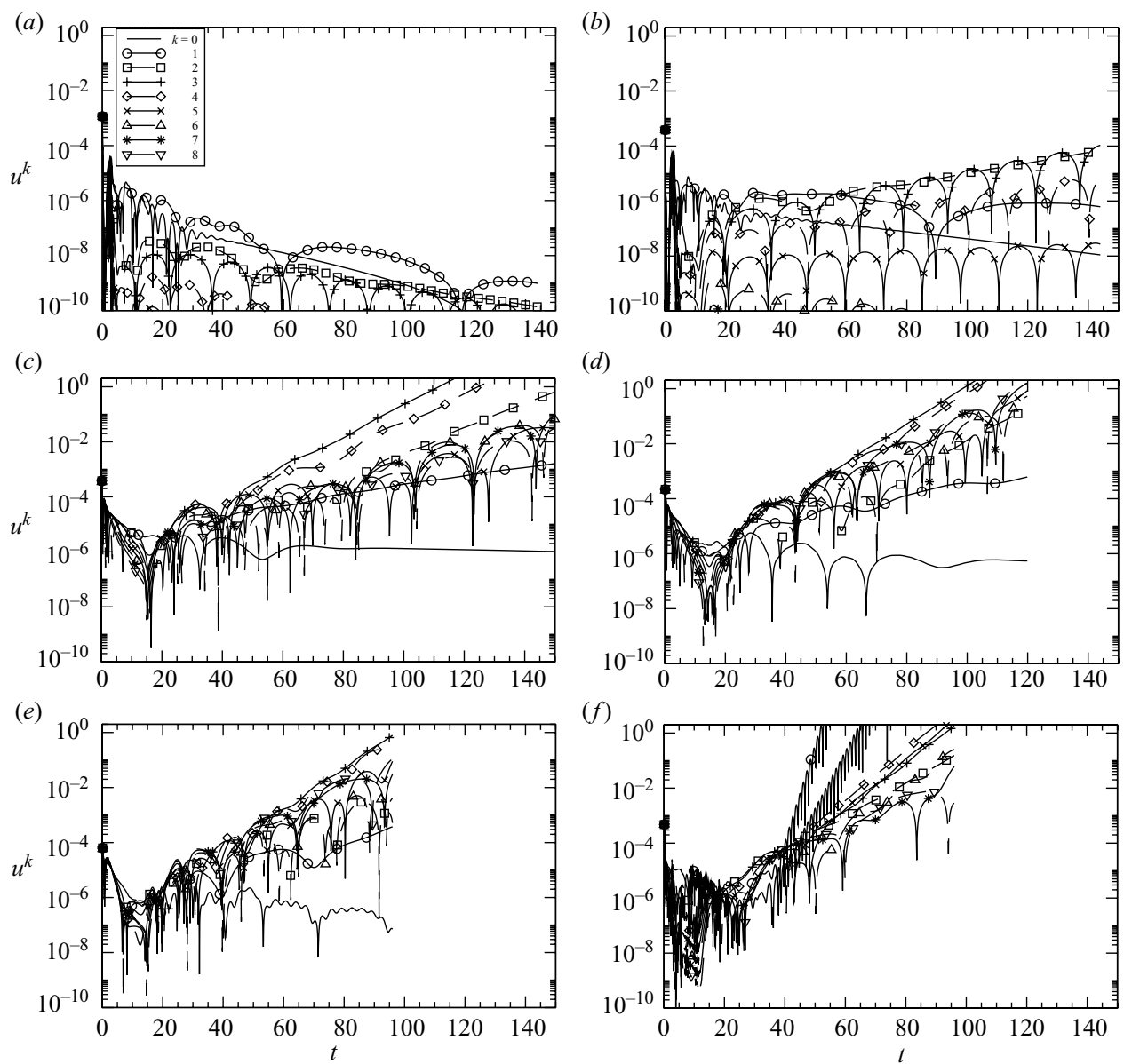

FIGURE 5. Temporal development of azimuthal Fourier modes of the streamwise velocity component obtained from LNS calculations for various Reynolds numbers; pulse location $z=2.5, r=0.5 ; M=2.46$. (a) $R e_{D}=5000 ;(b) 10000 ;(c) 30000 ;(d) 60000 ;(e) 100000$; (f) 200000 .

the axisymmetric mode exhibits a larger growth rate than all remaining modes. This confirms the suspicion, that, at this Reynolds number, an absolute instability of the axisymmetric mode could be present for the high-Reynolds-number conditions of the experiments by Dutton and co-workers.

To exclude the possibility that reflections from the domain boundaries introduced non-physical perturbations in the region of interest and continuously excited convective instabilities, the calculation for $R e_{D}=30000$ was repeated with a significantly larger domain size. The outflow boundary was chosen such that the flow was supersonic at each radial point to eliminate upstream-travelling reflections from the outflow boundary. The inflow of the computational domain was also moved to 2 radii upstream of the base. Furthermore, no perturbations from the free stream were anticipated for the radial domain size chosen. The Mach angle at $M=2.46$ is $\sim 24^{\circ}$, therefore, for a domain height of $r=6$, the expansion wave emanating from the base corner reaches the free stream at approximately $z=11$. Hence, reflections from the free-stream boundary will not interfere with the wake until well downstream 


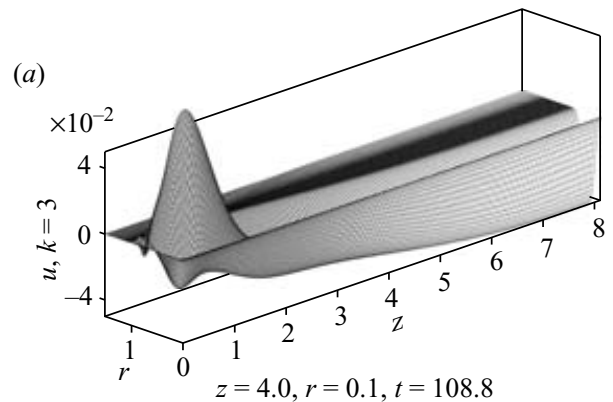

(b)
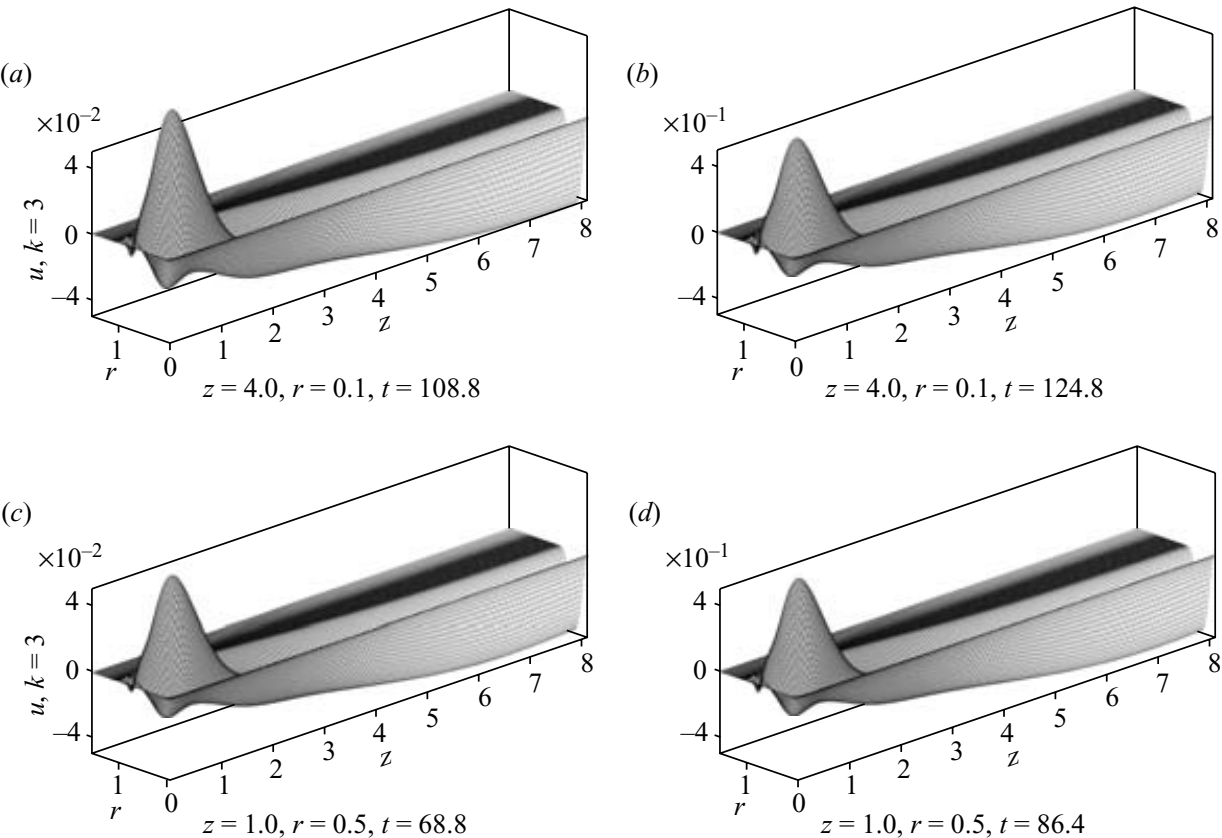

(d)
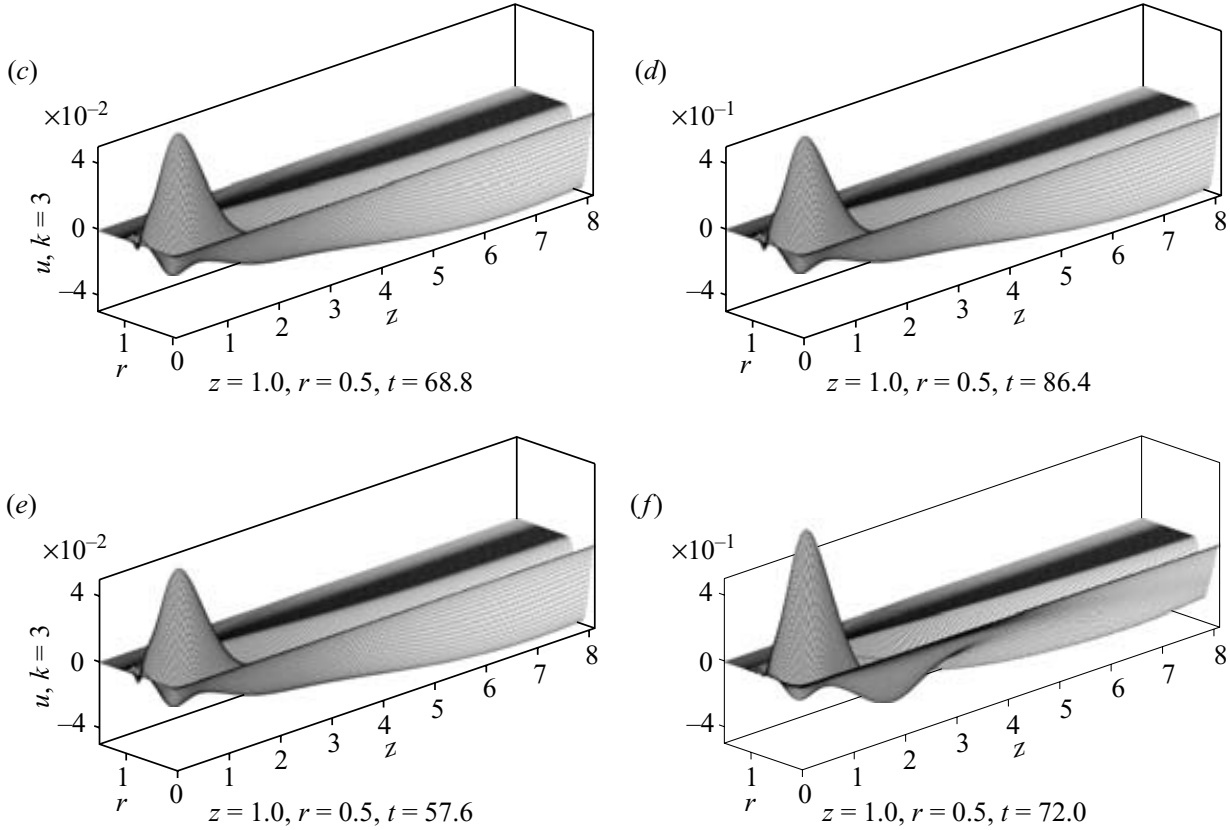

FIgURE 6. Two-dimensional mode shape of Fourier mode $k=3$ of the streamwise velocity component for different pulse locations obtained from LNS calculations; $(a-d) R e_{D}=30000$, $(e, f) R e_{D}=100000, M=2.46$.

of the potentially absolutely unstable region. The calculation with a different domain size showed no significant difference from the original computation, giving confidence in the results discussed above.

All LNS simulations were repeated inserting the initial pulse at different locations in order to investigate whether the resulting amplitude distribution is dependent on the initial condition. For all cases, once an initial transient is overcome, a two-dimensional amplitude distribution forms that remains unchanged in shape for increasing time. Representative of most cases, the mode shape of the third azimuthal Fourier mode of the streamwise velocity component is illustrated for various forcing locations at two instances each in figures $6(a)$ to $6(f)$. Comparing figure $6(c, d)$ with figure $6(a, b)$ shows no difference in the mode shape in spite of the disparate initial pulse locations. When inserting the initial disturbance at $z=1.0$ and $r=1.0$, i.e. into the shear layer, the same mode-shape is obtained, but with an opposite sign. The two instances shown for different forcing locations verify that even though the amplitude increases by an order of magnitude, the shape of the mode does not change. All simulations 
produced a local maximum close to the base. Scrutinizing the amplitude distribution at $R e_{D}=100000$, shown in figure $6(g, h)$, a fairly similar mode shape can be observed as for the lower Reynolds numbers with an additional local maximum close to the axis at $z \approx 2.0$. Again, for two sequential instances, the amplitude of the mode grows while the two-dimensional mode shape is maintained. It is also noteworthy that a similar two-dimensional mode shape was obtained from calculations at Reynolds numbers that were below the threshold of an absolute instability with respect to azimuthal modes. The presence of a two-dimensional mode arising independent of the pulse locations is an indication of the presence of a global mode. In order to verify that this might be the case, temporal calculations were conducted, which, owing to the parallel assumption of the basic state, inherently exclude the presence of global modes. The temporal results are presented in $\S 4.1 .3$.

Radial profiles of azimuthal Fourier modes for the most energetic (streamwise) velocity component, obtained from LNS calculations, are shown in figure 7 in order to establish the effect of Reynolds number on the amplitude distributions. A streamwise location within the recirculation region, $z=2.5$, was selected. For all data sets, the azimuthal modes were scaled with the global maximum of the first azimuthal Fourier mode. The amplitudes among separate Fourier modes were not considered crucial, as all modes are decoupled in the linearized calculations and, therefore, do not interact with each other.

For all Reynolds numbers investigated, the radial profiles of the axisymmetric mode $k=0$ possess their maximum in the vicinity of the shear layer. In contrast, for Reynolds numbers up to $R e_{D}=100000$, the amplitude distribution of the first azimuthal mode exhibits a global maximum at $r \approx 0.2$. For $R e_{D} \geqslant 200000$, however, the radial profiles exhibit an entirely different behaviour, with the global maximum of the first mode located in the shear layer. The above observations lead to the conclusion, that, in spite of being damped up to $R e_{D}=10000$ and amplified for $R e_{D} \geqslant 20000$, the shape of the first azimuthal mode is fairly independent of Reynolds numbers up to $R e_{D}=100000$ at $M=2.46$.

For azimuthal modes with $k>1$, a similar behaviour can be observed as for the first mode, i.e. for lower Reynolds numbers, the global maxima are located within the recirculation region and for increasing Reynolds numbers, an additional local maximum develops in the shear layer, becoming the absolute maxima for $R e_{D} \geqslant 200000$. As opposed to the first azimuthal mode, for higher modes the presence of a local maximum in the shear-layer region occurs for considerably smaller Reynolds numbers, starting at $R e_{D}=10000$.

This behaviour leads to the suggestion that two distinct instability mechanisms for the higher azimuthal modes might be present in the flow: A global mode within the recirculation region, and a convective shear-layer mode. The fact that the convective shear-layer mode becomes visible in the radial profiles only for higher Reynolds numbers is expected. The convective shear-layer mode is subject to strong damping in high-compressibility environments. A measure for the compressibility of the flow is the convective Mach number $M_{c}$, introduced by Bogdanoff (1983). When using the isentropic definition of $M_{c}$, the largest convective Mach number is reached at the streamwise location where the maximum reverse velocity can be found, i.e. in the initial shear layer. In fact, because of the rapid expansion at the base, the free-stream velocity also possesses its largest value at the same streamwise location. Therefore, large Reynolds numbers are required in order to enable the convective shear-layer instability to be amplified. Several azimuthal modes with $k>1$ display a strong contribution of the convective shear-layer mode at lower Reynolds numbers. This 
(a)

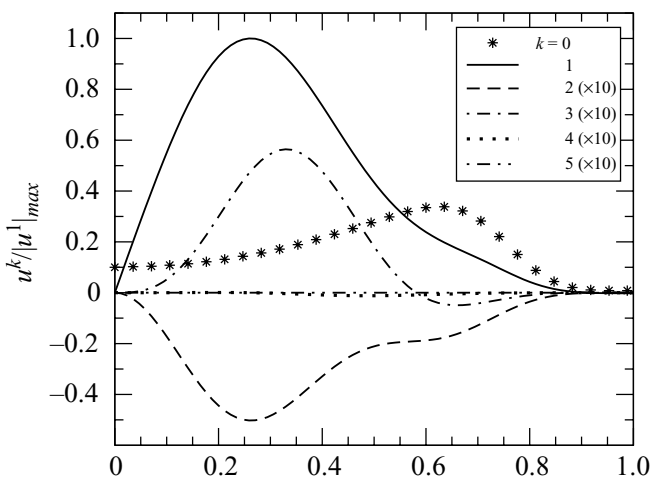

(c)

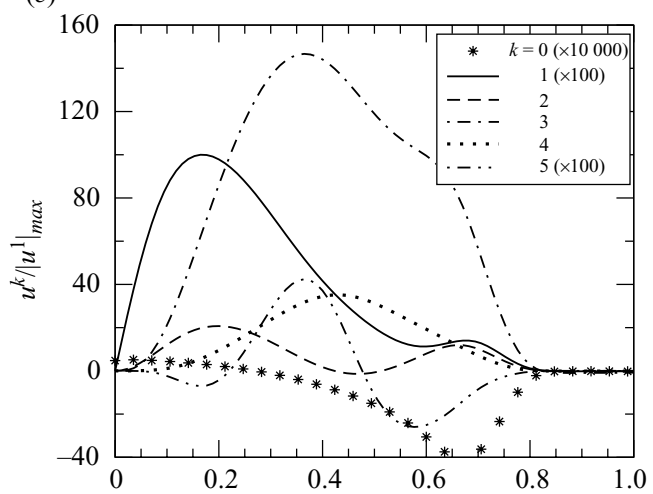

(e)

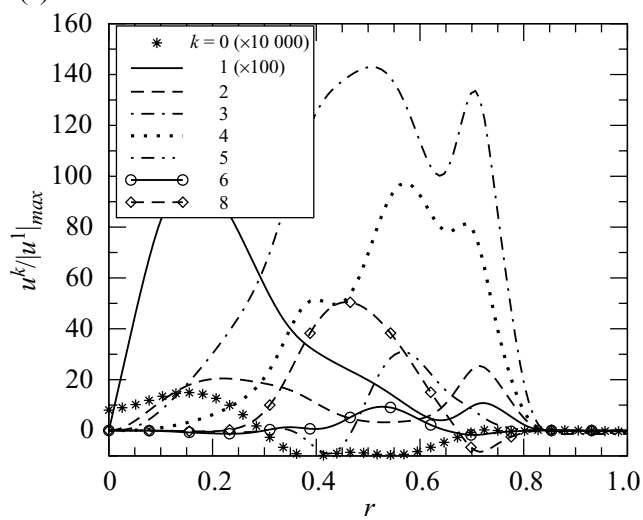

(b)

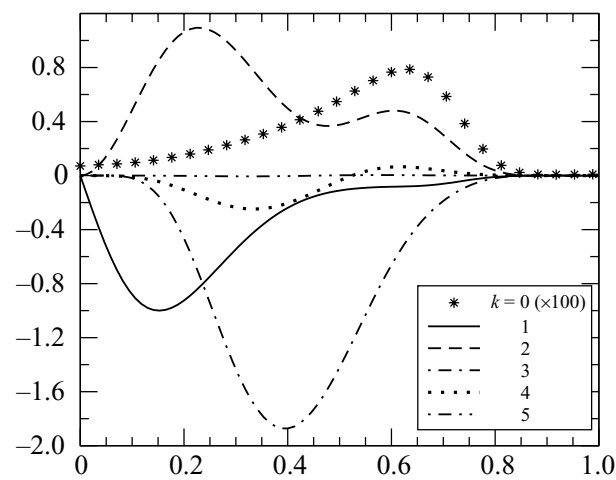

(d)

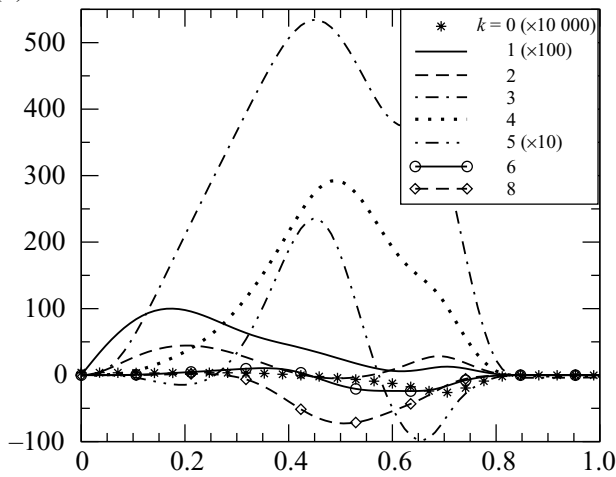

$(f)$

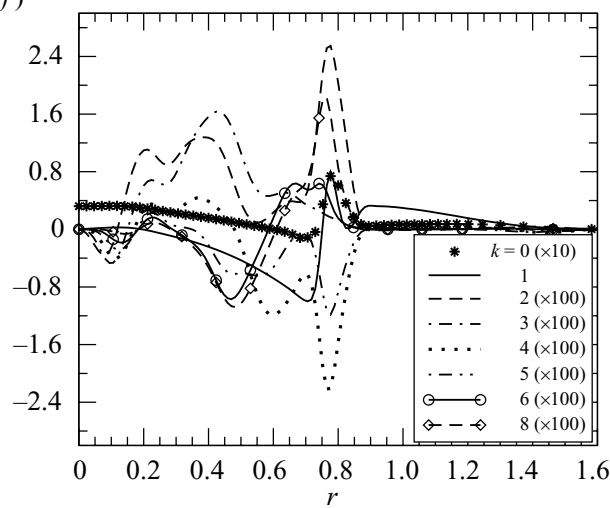

FIGURE 7. Radial mode shapes of Fourier modes of the streamwise velocity component, obtained from LNS calculations for Reynolds numbers: 5000, 10000, 30000, 60000, 100000 and 200000 from $(a)$ to $(f) ; z=2.5, M=2.46$.

can be attributed to the fact that oblique disturbances have larger amplification rates under high-compressibility conditions.

The location $z=2.5$ was selected for the above discussion because it is representative of a large part of the recirculation region. In light of the position of the local maxima of the two-dimensional mode shapes presented in figure 6, radial mode shapes were also scrutinized at the streamwise location $z=0.5$. In contrast to the location $z=2.5$, the global mode appeared to be dominant for all Reynolds numbers with merely a 
small contribution of the convective shear-layer mode visible in the data. The radial profiles of the azimuthal modes at a streamwise location downstream of the recompression region were also investigated. All mode shapes exhibited only one maximum, contracted to a smaller radial extent owing to the lesser circumference of the wake at the location further downstream. In the absence of a recirculation region, only the convective shear-layer mode was present at the location within the trailing wake.

From the spatial LNS simulations, circumstantial evidence was found for an absolute instability with respect to higher azimuthal modes, $k>0$, at $M=2.46$ for $R e_{D}>5000$. For $R e_{D}>100000$, the flow appears to be absolutely unstable with respect to the axisymmetric mode, $k=0$. For the Reynolds number range $10000 \leqslant R e_{D} \leqslant$ 100000 , the linearly most amplified modes are $k=2,3,4$. It is suggested that, for higher azimuthal modes, a global mode and a convective shear-layer mode co-exist. For increasing Reynolds numbers, the shear-layer mode gains in importance.

\subsubsection{Temporal results}

In order to determine whether the flow is locally or globally unstable with respect to axisymmetric or azimuthal/helical modes, stability investigations at various streamwise positions were conducted with the temporal code. Three positions were assumed to yield characteristic results. (i) A location fairly close to the base, at $z=0.5$. Here, the highest reverse velocity is reached for all Reynolds numbers investigated (see figure 4) and the shear layer features a large radial gradient just downstream of separation. In the spatial calculations, the maximum of the global mode was found at this position. (ii) A position farther downstream, at $z=2.5$, was chosen, where the shear layer has grown and the radial gradient is reduced. This location is representative of a large area within the recirculation region. (iii) A location downstream of the reattachment point, at $z=7$ or $z=9$, was selected. No reverse flow is present within the trailing wake; however, a significant velocity deficit occurs. The same numerical grid in the radial direction was used in the temporal case as for the spatial calculations. The azimuthal extent of the domain was $0 \leqslant \theta \leqslant \pi$ for all cases. However, which streamwise length of the domain to choose was not obvious. Therefore, several calculations were conducted with a fairly long streamwise extent $\left(\lambda_{z}=4 \pi\right)$ and a large number of streamwise Fourier modes $(l=32)$. The calculations revealed that no modes with $l \leqslant 2$ or $l \geqslant 12$ were strongly amplified. Thus, all stability computations were done with 8 streamwise modes for a domain length of $\lambda_{z}=2 \pi$. An initial pulse was introduced at the inflection point of the respective profile and the development of the streamwise and azimuthal Fourier modes was monitored at the forcing location.

Results for Reynolds numbers 5000, 30000 and 100000 are shown in figure 8 . For clarity, only the most amplified Fourier modes are shown. Recall that the mode numbers are given in the format $(l, k)$, where $l$ denotes the streamwise mode number and $k$ denotes the azimuthal wavenumber. Each azimuthal mode is assigned one symbol and the streamwise modes are distinguished through separate line styles. In general, it can be observed that the growth rates of the most amplified modes increase drastically with increasing Reynolds numbers. Also, the closer the location of the profile under investigation is to the base, the higher the growth rates appear to be and the more modes experience strong amplification. The graphs also reveal that the most unstable modes are always helical (oblique) modes, i.e. $k, l \neq 0$. In fact, unstable axisymmetric modes $(k=0)$ are only found for the two upstream locations, and no unstable streamwise modes $(l=0)$ were found at all in the linear regime. 
(a)

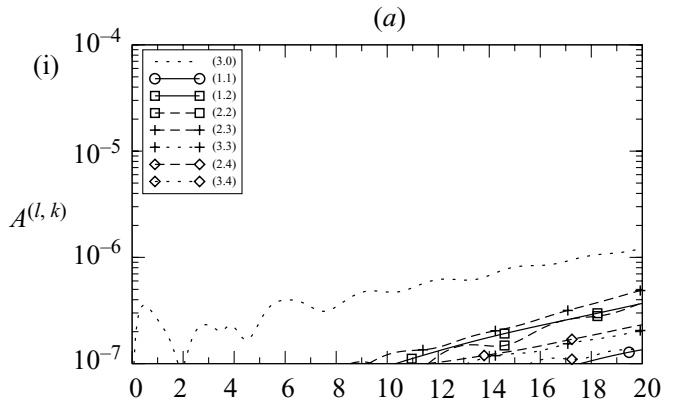

(ii)

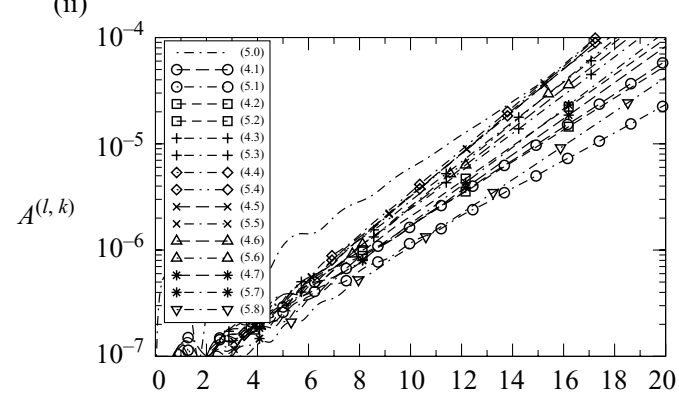

(iii)

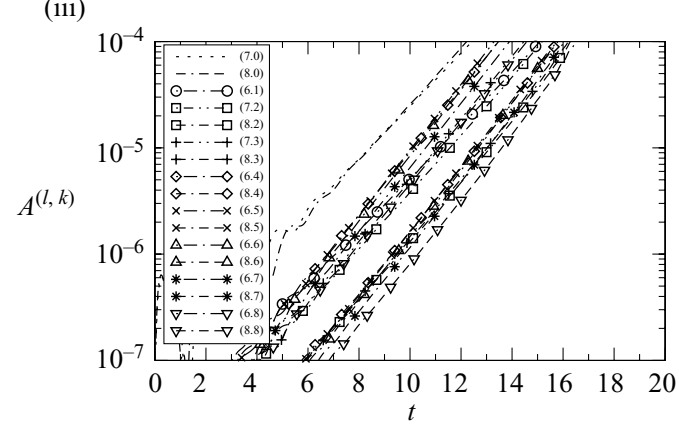

(b)
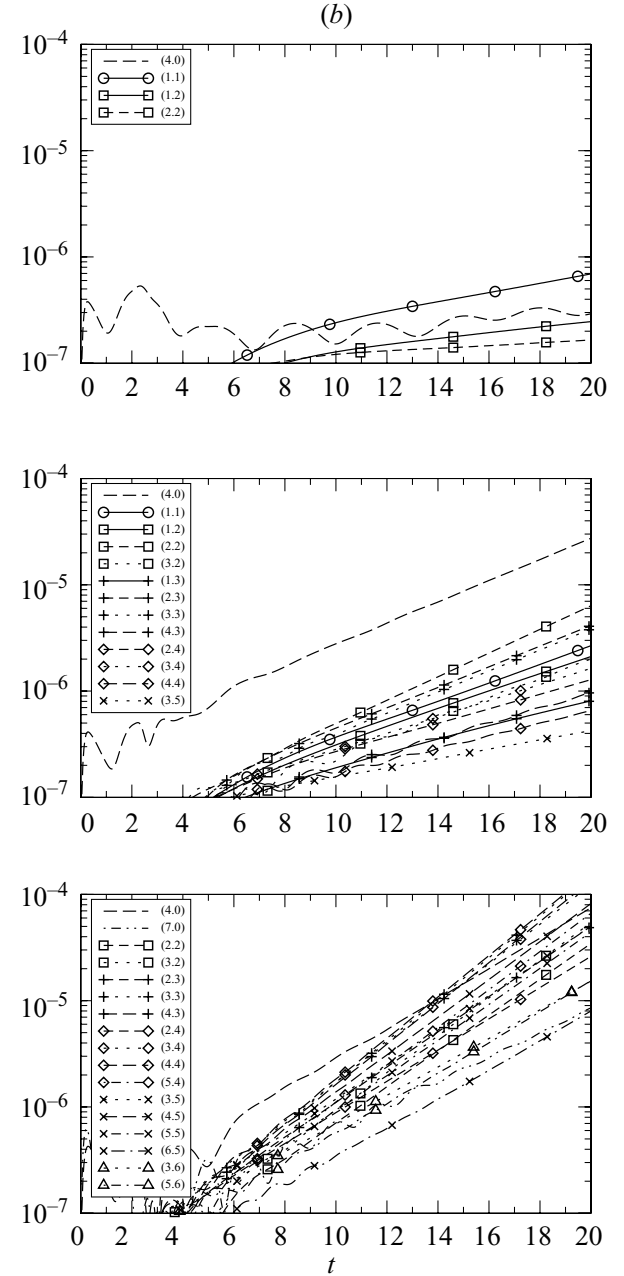

(c)
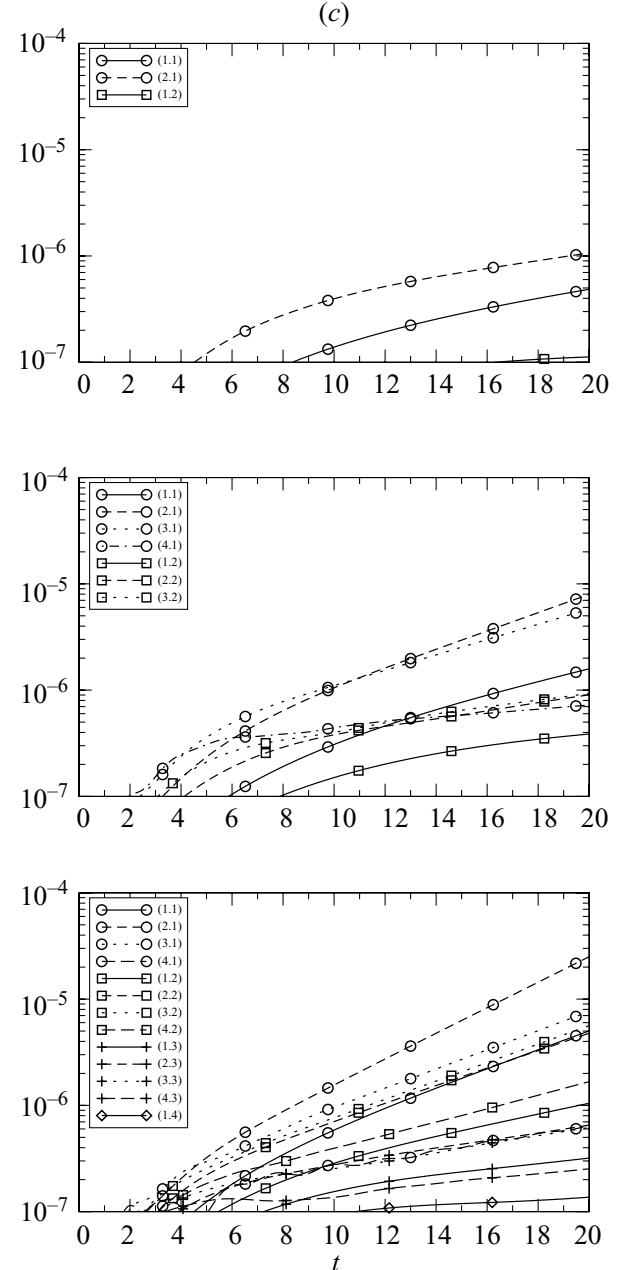

FIGURE 8. Temporal development of Fourier modes of density obtained from TDNS for (i) $R e_{D}=5000$, (ii) $R e_{D}=30000$ and (iii) $R e_{D}=100000$; (a) $z=0.5$, (b) $z=2.5$ (c)(i) $z=9$, (c)(ii)(iii) $z=7 ; M=2.46$. 
(a)

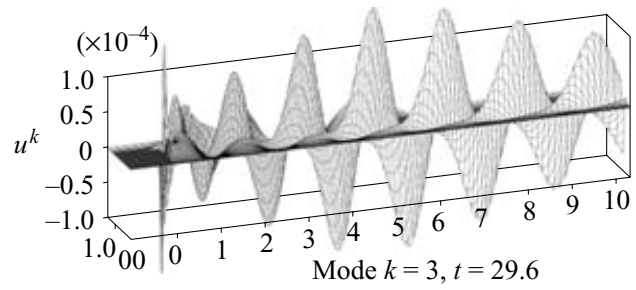

(b)

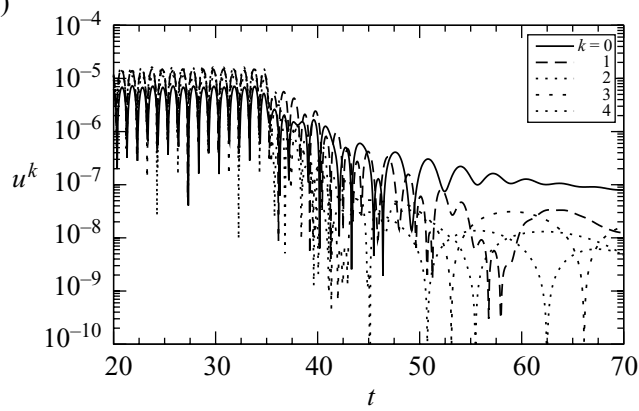

FIGURE 9. Instantaneous azimuthal Fourier mode of $u$ with periodic forcing active $(a)$ and (b) temporal development of azimuthal Fourier modes of $u$ obtained from an LNS calculation employing periodic forcing; $R e_{D}=5000, M=2.46$.

For the lowest Reynolds number investigated (5000), at $z=0.5$, modes with $k=2,3,4$ are most amplified with the streamwise mode numbers being $l \leqslant 3$. Further downstream, at $z=2.5$, where the reverse velocity is reduced and the radial shearlayer gradient is diminished, only the first two azimuthal modes are amplified for long streamwise wavelengths. Within the trailing wake, the local analysis shows that only the first azimuthal mode is unstable for $l=1,2$. Increasing the Reynolds number, a trend, that larger azimuthal mode numbers also become strongly amplified, can be established. However, a significant difference can be observed when comparing the three streamwise locations. For the location within the trailing wake, the streamwise mode number of the most unstable modes does not increase considerably, i.e. $l=2,3$ for all $k$. In contrast, at $z=0.5$, the streamwise wavenumber of the most amplified Fourier modes increases steadily with Reynolds number such that, at $R e_{D}=100000$, modes with $l \geqslant 4$ are most amplified for all $k$. The location at $z=2.5$ shows a similar behaviour, however, the shift towards higher streamwise wavenumbers is not as pronounced. In order to assert that no streamwise modes higher than $l=8$ were significant, the calculations at $z=0.5$ for the higher Reynolds numbers were repeated with a streamwise domain length eight times smaller, i.e. $\lambda_{z}=0.25 \pi$. For these cases, $l=1$ corresponds to $l=8$ in the calculations with the longer domain length. The additional calculations revealed that no modes with $l>8$ (in the original case) were strongly amplified for the Reynolds numbers investigated.

In contrast to the temporal case, in the spatial calculations, the flow showed no signs of being unstable with regard to higher azimuthal modes at $R e_{D}=5000$. Owing to the periodic boundary conditions in the streamwise direction, the temporal approach converts every physical problem to a closed system, i.e. disturbances cannot leave the system. If the flow is convectively unstable, the disturbance will, therefore, grow in time, which in the spatial case would be defined as an absolute instability. In order to verify that the instabilities detected for $R e_{D}=5000$ in the temporal code are of a convective nature, i.e. require continuous excitation in order to remain in the region of interest, an additional spatial calculation was conducted. Here, a time-periodic volume forcing was introduced in the shear layer immediately after separation (centre of disturbance at $r=1.05, z=0.04$ ). The periodic forcing at a frequency of $\omega=0.5$, which appeared to be the most amplified one in the temporal calculations, was active for the time-interval $0 \leqslant t \leqslant 32$. The forcing was then switched off, in order to evaluate whether the disturbances were entirely convected out of the computational domain. The result for the third azimuthal mode of $u$ is shown in figure 9. 


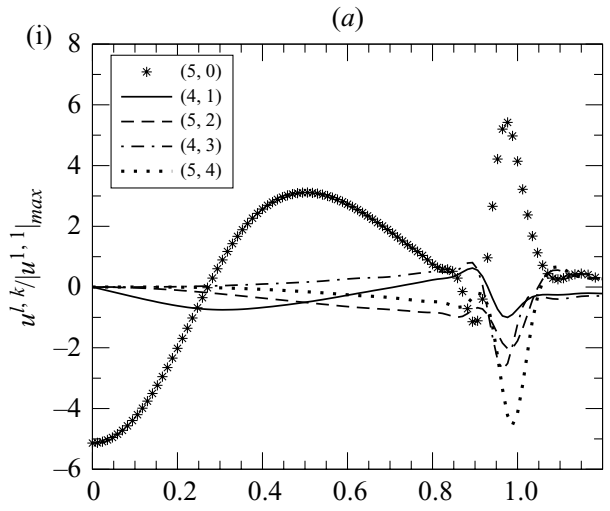

(i)
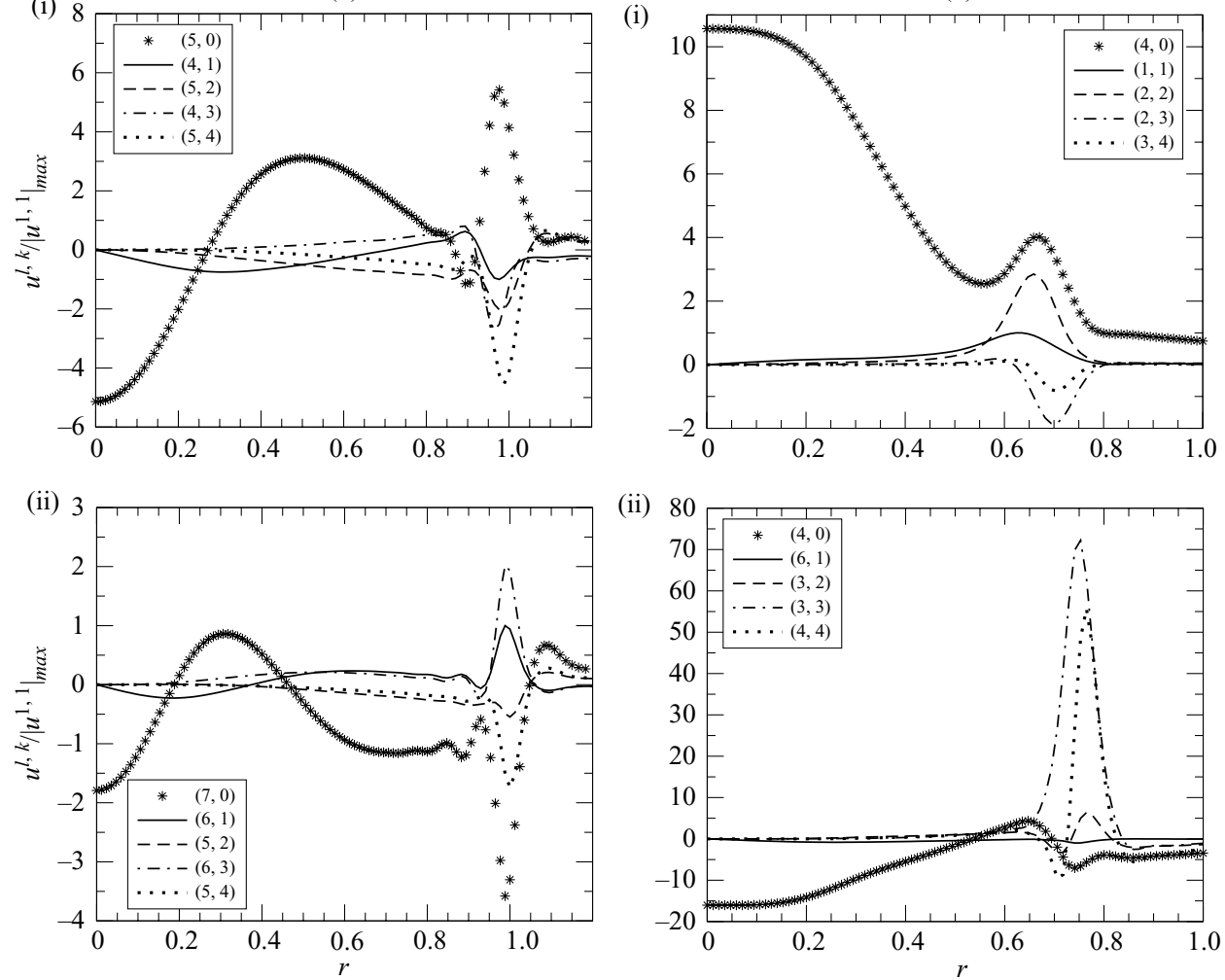

FIGURE 10. Radial mode-shapes of Fourier modes of the streamwise velocity component, obtained from TDNS; (a) $z=0.5$, (b) $z=2.5$; (i) $R e_{D}=30000$, (ii) $R e_{D}=100000 ; M=2.46$.

At $t=29.6$, while the forcing is still active, it can be observed that the disturbances grow in the streamwise direction up to the end of the recirculation region (the recirculation length of the basic state is $z=4.4$ ) and then start to decay. Thus, the disturbances experience a spatial amplification. At $t=41.6$, the forcing was switched off and the disturbances are convected downstream. At a later time (not shown here), no disturbances remain in the region of interest. To verify that the disturbances contained in the recirculation region truly decay once the forcing is switched off, the temporal development of the Fourier modes is also shown in figure 9. While the forcing is still active, no temporal growth can be observed at the probe point. All modes exhibit an oscillation with the forcing frequency and the amplitude being similar to the forcing amplitude. After switching off the periodic forcing, all modes start decaying $(t \approx 34)$ once the last disturbance generated at the forcing location $(r=1.05, z=0.04)$ has passed the probe point $(r=0.5, z=2.5)$. This behaviour is a clear indication that, for this Reynolds number, the flow is convectively unstable (and not absolutely unstable) with regard to the higher azimuthal modes.

For all Reynolds numbers and locations $z \geqslant 2.5$ investigated with the temporal code, the radial mode shapes exhibit a maximum exclusively in the shear-layer region. As shown in figure $10(b)$, virtually no variation can be observed within the recirculation region, implying that, at this location the local analysis only captures the convective shear-layer mode. This supports the conjecture that the inner maximum found in the radial profiles obtained from spatial calculations might indeed be caused by 
global modes. The above results also substantiate the view that the convective shearlayer instability is generated locally. Non-zero phase speeds found for all unstable modes confirm that the shear-layer mode is a convective instability. However, at the streamwise location $z=0.5$, which corresponds to the maximum of the twodimensional mode shape shown in figure 6 , a considerable variation within the recirculation region can be observed (see figure 10a). It is suggested that this is a consequence of the TDNS capturing a local absolute instability at this location. This phenomenon was observed for the region $0.1 \lesssim z \lesssim 1$. According to Huerre \& Monkewitz (1990), a region of local absolute instabilities is the necessary condition for a global mode. Therefore it is suggested that the temporal results constitute further evidence for the presence of global modes.

Radial profiles obtained from local stability calculations using profiles from the trailing wake (not shown here) showed a similar behaviour to the spatial results. This supports the notion that the trailing wake region contains only the convective shear-layer mode.

Summarizing the results obtained from TDNS calculations, for all Reynolds numbers and streamwise positions investigated, unstable helical/oblique modes were found. For $z \gtrsim 1$, only convectively unstable shear-layer modes were detected, supporting the view that the shear-layer modes are generated locally. For streamwise locations closer to the base, it appears as if the basic state becomes absolutely unstable, satisfying the necessary condition for a global mode.

\subsection{Direct numerical simulations}

The results presented in the previous subsection have given valuable insight into the linear stability characteristics of supersonic axisymmetric wakes at various Reynolds numbers. Nevertheless, by excluding nonlinear effects, the occurrence of coherent structures and the breakdown to turbulence is not permitted. The main objective of this work is, however, to investigate the formation of coherent structures that are a consequence of physical flow instabilities. To that end, DNS were employed to investigate the near-wake region in supersonic axisymmetric wakes. It was of particular interest to determine the significance of certain modes and their effect on the mean flow. This was accomplished by exploiting one of the strengths of conducting numerical simulations: deliberately simplifying the physical problem at hand. Here, simulations were conducted for various circumferential domain sizes, thereby intentionally eliminating different azimuthal/helical modes. Thus, the effect of large-scale structures associated with particular azimuthal/helical modes on the global flow behaviour could be evaluated.

For all Reynolds numbers investigated in this work, initially a DNS for a halfcylinder (domain with $0 \leqslant \theta \leqslant \pi$, due to the use of symmetric Fourier transforms) was conducted. The DNS for $R e_{D}=30000$ was repeated on a separate computational grid and it was shown that the solution was independent of the grid-resolution (see Sandberg \& Fasel 2006). In addition, the turbulent dissipation rate was evaluated in all simulations and used to calculate the Kolmogorov length in the entire integration domain. It was found that the local grid spacing was fine enough to resolve all relevant length scales (cf. Sandberg \& Fasel 2006). Once it was verified that the grid resolution was adequate, simulations of integration domains with an azimuthal extent of $\theta<\pi$ were also conducted, deliberately suppressing various modes of the half-cylinder case. Note that in the following, the mode numbers denoted by $k$ refer to the corresponding positive and negative mode numbers in the half-cylinder case, not the computational mode of the respective calculation, i.e. mode $k=2$ always has a wavelength of $\pi$, for example. The DNS calculations at $M=2.46$ were conducted 
for several Reynolds numbers. The lowest Reynolds number investigated was $R e_{D}=$ 30000 . Here, the computational domain was discretized using $516 \times 200$ points in the streamwise and the radial direction, respectively, with the smallest grid spacing at the corner, where $\Delta z_{c}=\Delta r_{c}=0.008$. In the azimuthal direction, the simulation of the half-cylinder case was carried out with 65 symmetric spectral modes. The number of Fourier modes for the calculations with smaller circumferential domain sizes was chosen such that the azimuthal resolution remained constant, i.e. 33, 25, 17 and 9 modes were used for the quarter-cylinder, 1/6-cylinder, 1/8-cylinder and the 1/16-cylinder calculations, respectively.

The largest Reynolds number chosen was $R e_{D}=100000$, as this constituted the highest Reynolds number possible with the resources available. DNS of a half-cylinder, a quarter-cylinder, $1 / 8$ and $1 / 16$ of a cylinder were conducted. All calculations were performed on the same streamwise/radial grid with $1272 \times 160$ points in the streamwise and the radial directions, respectively. The smallest grid spacing at the corner was $\Delta z_{c}=\Delta r_{c}=0.008$. In the azimuthal direction, the simulation of the half-cylinder was conducted with 129 symmetric spectral modes; for the cases with smaller circumferential extents, 65, 33, 25 and 17 Fourier modes were used. For all cases, the approach flow was laminar, and transition to turbulence occurred downstream of the separation point.

The response of the higher azimuthal density modes to the initial pulse was monitored at the disturbance location for all cases investigated. Rapid growth was observed for all domain sizes and Reynolds numbers until a nonlinear saturation state was attained. A discussion of the temporal development of the azimuthal Fourier modes and a temporal Fourier analysis was presented in Sandberg \& Fasel (2006). The authors found that the flow is strongly intermittent up to $R e_{D}=30000$, but shows no sign of intermittency for larger Reynolds numbers. Temporal spectra revealed peaks with low frequencies for the small-wavenumber modes in all cases, suggesting the presence of large-scale structures. For Reynolds numbers exceeding 30000 , a considerable amount of energy was also found in frequencies with $S t_{D} \geqslant 1$. It was demonstrated that dominant peaks in the temporal spectra of the axisymmetric mode $k=0$ could be attributed to the nonlinear interaction of the most important helical modes with themselves or $k=0$. For small circumferential domain sizes, a viscous cutoff of the high wavenumbers was shown to prevent the high azimuthal modes from growing linearly. In addition, because the long wavelengths are eliminated in these cases, the large wavenumbers could not be generated nonlinearly either. Consequently, no small-scale structures were generated in these cases. In the current discussion, the main focus will be on coherent structures generated by the flow instabilities and their effect on the mean flow.

\subsubsection{Coherent structures}

In order to identify what kind of structures are generated in the flows under investigation and how those structures evolve, a vortex-identification method was employed. Terzi, Sandberg \& Fasel (2006) performed a study of various vortex eduction methods and concluded that the $Q$-criterion is an adequate choice even for compressible flows as the desired information can be obtained from available data at low computational cost and the method can be implemented easily. The $Q$-criterion is named after the second invariant of the gradient of the velocity vector and for compressible flows is computed as

$$
Q=0.5\left[\left(\frac{\partial u_{i}}{\partial x_{i}}\right)^{2}+W_{i k} W_{i k}-S_{i k} S_{i k}\right]
$$


(a)
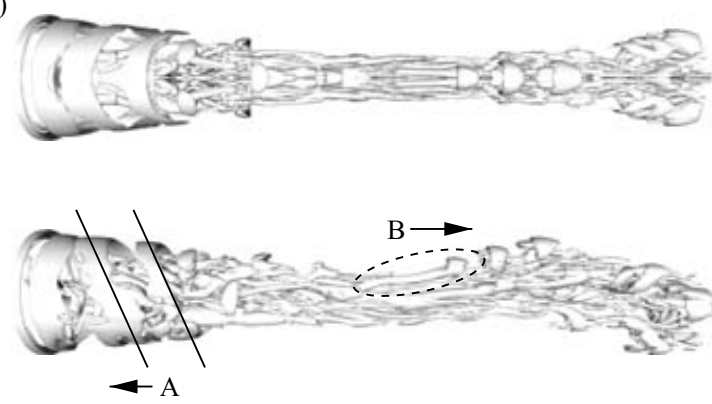

(b)

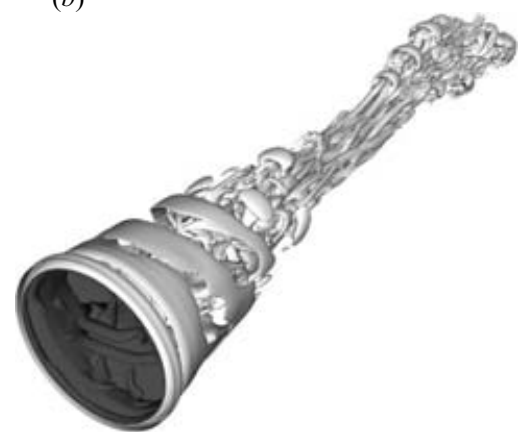

Figure 11. Instantaneous iso-contours of $Q=0.1$ for half-cylinder case, $(a)$ top- and sideview (top and bottom) and (b) perspective view from inflow towards outflow; $R e_{D}=30000$, $M=2.46$.

with

$$
S_{i k}=0.5\left(\frac{\partial u_{i}}{\partial x_{k}}+\frac{\partial u_{k}}{\partial x_{i}}\right), \quad W_{i k}=0.5\left(\frac{\partial u_{i}}{\partial x_{k}}-\frac{\partial u_{k}}{\partial x_{i}}\right) .
$$

Figure 11 shows several views of instantaneous iso-contours of $Q=0.1$ for the half-cylinder case at $R e_{D}=30000$. In figure 11(a), top- and sideviews are shown and in figure $11(b)$ a perspective view, looking from the inflow towards the outflow, is visualized with the dark-grey surface marking the base. Comparing the visualizations of $Q$ for the supersonic case with results obtained for subsonic wakes (see, e.g. Hannemann \& Oertel 1989; Huerre \& Monkewitz 1990; Schwarz et al. 1994), the considerable difference in Mach number appears to lead to a fundamentally different flow behaviour. In the subsonic case, large vortex loops are shed alternately on the upper and lower side, highlighting the dominance of the first helical mode. In contrast, in the current supersonic case, close to the base, oblique, or helical, structures can be seen in the initial shear layer, denoted by 'A'. Because of the large convective Mach number $M_{c}$ in this region, the generation of oblique, or in this case, helical disturbances can be expected. Also, the local analysis using temporal simulations showed that the unstable local convective shear-layer modes are of a helical nature with $k, l \neq 0$. This implies that the helical structures in the shear layer are a consequence of the local convective shear-layer mode. In addition, longitudinal structures are present within the recirculation region that protrude into the trailing wake.

Farther downstream, the convective Mach number decreases (as the magnitude of reverse velocity in the recirculation region decreases) and mainly longitudinal structures and several vortex loops appear. The structures observed within the trailing wake strongly resemble hairpin structures, commonly observed in boundary-layer transition (one of them marked with 'B'). The 'legs' of these structures can be seen to extend into the recirculation region and the 'heads' of the hairpin vortices form vortex loops which travel downstream and move away from the axis. Similar to the boundary-layer transition scenario, once the 'heads' of the hairpin vortices are lifted, an instantaneous layer of high shear is developed, which is highly unstable and is the basis for further instabilities that generate high-frequency oscillations. This process appears to be one of the mechanisms responsible for the generation of small-scale structures that can be observed farther downstream in the trailing wake.

The helical structures appearing within the shear layer exhibit a sinusoidal modulation with a fairly large wavelength, implying that azimuthal modes with a small 
(a)

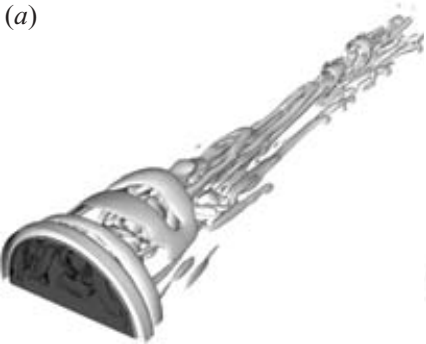

(b)

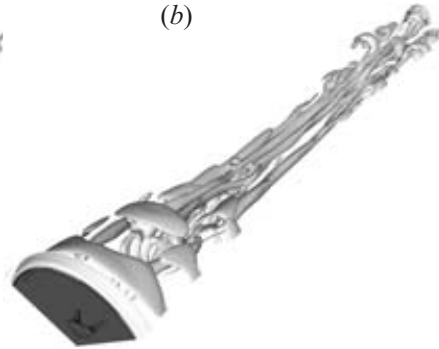

(c)

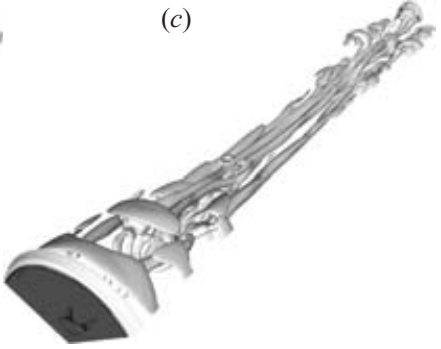

FiguRE 12. Instantaneous iso-contours of $Q=0.1$; perspective view from inflow towards outflow of (a) quarter-cylinder, (b) 1/6-cylinder and (c) 1/8-cylinder cases; $R e_{D}=30000$, $M=2.46$.

wavenumber are significant. In addition, the lateral motion (flapping) of the entire wake in the $(r, z)$-plane at $\theta=0, \pi$ can also be observed in figure 11, particularly in the sideview, although not as pronounced as for incompressible flows. This suggests that the first azimuthal mode plays a considerable role.

In figure 12, perspective views of iso-contours of $Q=0.1$ are presented for cases with a smaller azimuthal extent. Even though the low wavenumber modes are excluded, qualitatively, the same flow features can be observed as for the half-cylinder case, i.e. helical structures within the shear layer and hairpin vortices with 'legs' extending from the trailing wake, into the recirculation region. However, when visualizing larger values of $Q$ (not shown here), considerably fewer small-scale structures are visible than for the half-cylinder case. This is an indication that the modes with low wavenumbers are partly responsible for the creation of small-scale structures, either directly or through the generation of further instabilities.

For all cases shown so far, a large number of longitudinal structures was not only seen in the trailing wake, but also within the recirculation region. An effort is now made to unveil how these vortices are generated. One possible mechanism is proposed here: on the basis of linear stability calculations (see §4.1) it was suggested that the flow is absolutely unstable with respect to global modes within the recirculation region. Once the amplitudes of these global modes reach sufficiently large values, chevron-like patterns are imposed onto the recirculating fluid. These patterns are similar to those illustrated in figure 18. For a fully developed recirculation region, most probably multiple mechanisms compete, complicating the reconstruction of the most dominant events. For that reason, a time-sequence of the initial start-up of the $1 / 8$-cylinder calculation is shown in figure 13 in order to isolate individual events. Iso-contours of $Q=0.4$ are shown in a perspective view, looking from downstream towards the base.

Initially, the amplitudes of the higher azimuthal modes are small and the flow is fully axisymmetric, with an axisymmetric structure 'A' generated by the stagnation point flow at the base (marked by a black surface). In the second figure of the timesequence, a significant azimuthal modulation of the axisymmetric structure can be observed (' $\mathrm{B}$ '). The circumferential variation occurs within the recirculation region and not in the shear layer. Linear stability calculations at $R e_{D}=30000$ (figure 7) showed that mode $k=4$, which is the fundamental azimuthal mode for the 1/8-cylinder case, possesses the largest amplitude at $r \approx 0.45$ and was, therefore, classified as a global mode. Consequently, it can be speculated that the global modes are responsible for the azimuthal modulation of structure A. This sinusoidal variation in the circumferential direction appears to be the cause for the development of longitudinal structures, 

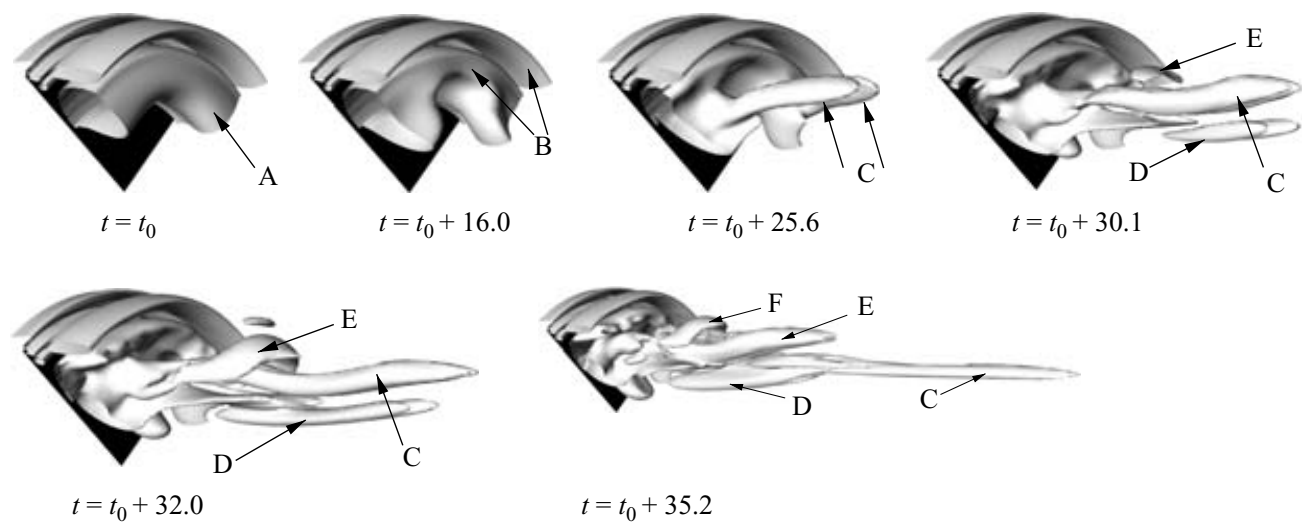

$t=t_{0}+32.0$

$t=t_{0}+35.2$

FIGURE 13. Time-sequence of instantaneous iso-contours of $Q=0.4$ for initial transient of $1 / 8$-cylinder case; perspective view from the trailing wake towards the base; $\operatorname{Re}_{D}=30000$, $M=2.46$.

visible at the next time instant, $t=t_{0}+25.6$, and marked with ' $\mathrm{C}$ '. In the following graph, it can be seen that another streamwise structure has been generated ('D'), which pushes $C$ toward the shear layer through induction of radial velocity. The structure $\mathrm{C}$ reaches the part of the shear layer where the streamwise velocity component is positive and the outer part of $\mathrm{C}$ is convected downstream, thereby stretching the vortex. Another structure, similar to $\mathrm{C}$, is present, albeit the iso-value at this time instant is smaller than that of $\mathrm{C}$ and, therefore, only the outermost part can be seen, denoted by 'E'. Going further in time, to $t=t_{0}+32$, it can be observed that the outer parts of $\mathrm{C}$ and $\mathrm{E}$ have travelled downstream and the structures have been stretched in the streamwise direction. In the last time instant shown, several observations can be made. The outer part of $\mathrm{C}$ appears to have overcome the adverse pressure gradient in the recompression region and has travelled a considerable distance downstream. $\mathrm{D}$, on the other hand, remains fully within the recirculation region and actually travels slowly upstream again. The structure $\mathrm{E}$ follows $\mathrm{C}$ and the next structure, marked ' $F$ ', appears. Once the initial transient is overcome, the recirculation region continuously contains several streamwise structures and, in addition, vortex loops can be observed (see, e.g. the structures at the base seen in the perspective view of the half-cylinder case in figure 11). These structures constantly impinge on the shear layer and thereby introduce disturbances. Because the shear layer was shown to be convectively unstable, the perturbations experience amplification in the streamwise direction which most probably results in the generation of additional structures.

To support the observation that the longitudinal structures within the recirculation region are generated when global modes reach large amplitudes, additional temporal calculations were performed. The local stability calculations conducted with the temporal code, presented in $\S 4.1 .3$ were continued into the nonlinear regime. The resulting flow fields obtained from the temporal simulations at several streamwise locations are shown in figure 14. At the location close to the base, the occurrence of helical structures in the shear layer, as seen in the spatial calculations, is clearly visible. When the temporal calculation is conducted using the local profile at $z=2.5$, several large vortex loops develop which are most probably a consequence of the nonlinearly strongly amplified modes $k=4$ and $k=6$. However, for both locations, no streamwise structures can be observed within the recirculation region. This reinforces the suggestion that the longitudinal structures are a consequence of additional 

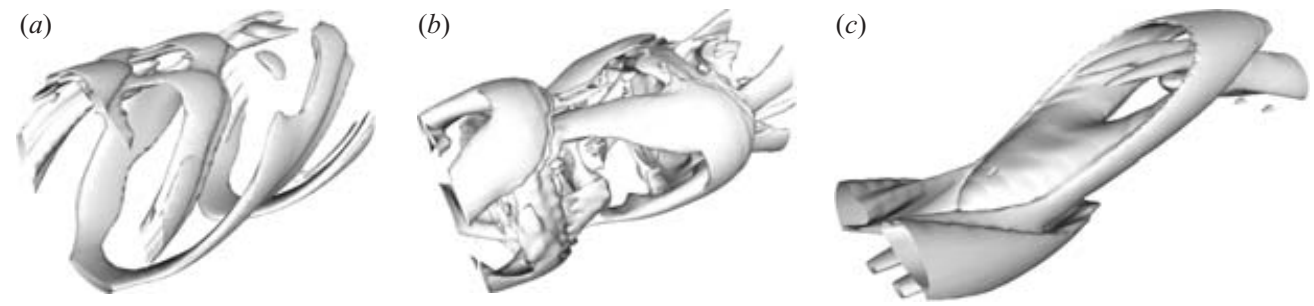

Figure 14. Perspective views of iso-contours of $Q=0.1$ from temporal calculations of half-cylinder case, $(a) z=0.5,(b) z=2.5$ and $(c) z=9 ; R e_{D}=30000, M=2.46$.
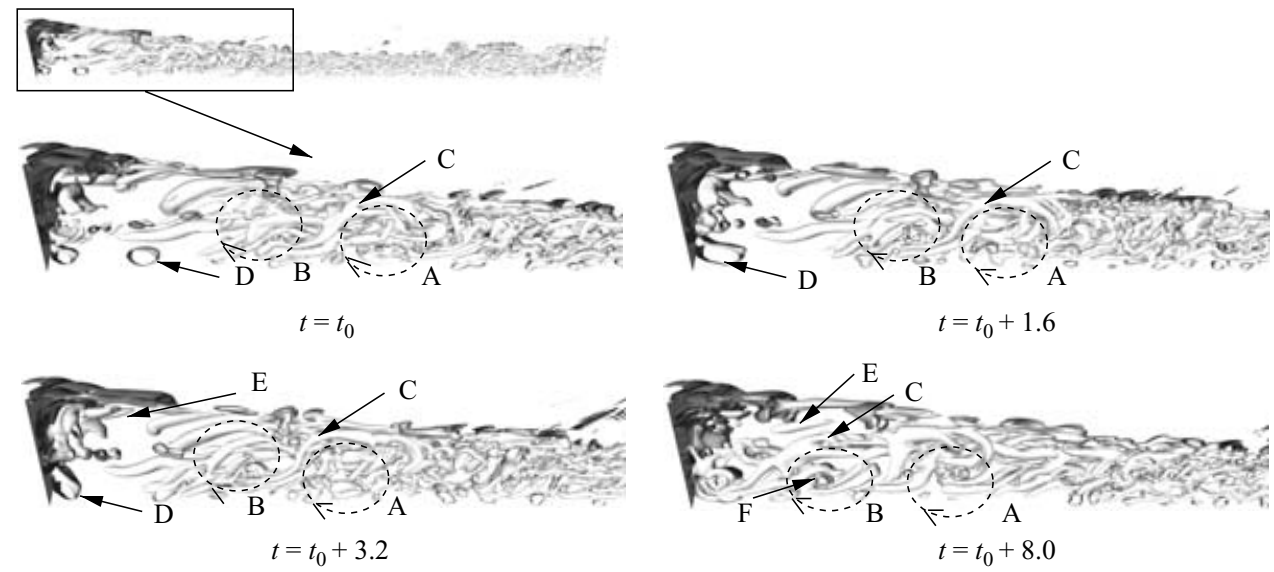

FIGURE 15. Instantaneous iso-contours of $Q=0.1$ for $1 / 16$-cylinder case; time-sequence of sideviews; $\operatorname{Re}_{D}=100000, M=2.46$.

instabilities occurring when global modes within the recirculation region reach finite amplitudes. Looking at the flow field obtained from a temporal simulation using a profile from the trailing wake, large vortex loops, similar to those observed in subsonic wakes, can be seen. These vortices are a consequence of modes $k=2$ and $k=4$ reaching a nonlinear saturation level. It can be speculated that in the presence of streamwise pressure gradients, significant stretching of these structures would occur, which might lead to similar structures to those observed in the spatial case. Therefore, it can be assumed that the hairpin vortices in the trailing wake might be partly generated locally. The results obtained from the temporal simulations suggest that the helical structures in the shear layer and the hairpin structures in the trailing wake, observed in the spatial calculations, are a consequence of local instabilities and are not caused by global effects. In contrast, because the longitudinal structures within the recirculation region could not be reproduced with local calculations, it is conjectured that these structures are most probably a consequence of a global instability.

In addition to the above described mechanism, the data extracted from simulations conducted at $R e_{D}=60000$ and $R e_{D}=100000$ suggest that an additional mechanism might be responsible for the generation of longitudinal structures within the recirculation region: the structures might possibly be a consequence of centrifugal instabilities (as introduced by Rayleigh 1916) that arise in the presence of strong instantaneous streamline-curvature. The time-sequence for the $1 / 16$-cylinder case at $R e_{D}=100000$ is shown in figure 15 to support this conjecture. A pair of axisymmetric rollers, that are composed of several smaller-scale structures, are present in the recirculation region 
at $t=t_{0}$ and are denoted by 'A' and ' $\mathrm{B}$ '. The streamwise structure (' $\mathrm{C}$ ') has strong similarity with structures observed in LES of a mixing layer by Lesieur \& Metais (1996). It is most probably formed as a consequence of a centrifugal instability, caused by strong instantaneous streamline curvature. Furthermore, an axisymmetric structure, denoted by ' $\mathrm{D}$ ' is positioned close to the axis, roughly one radius downstream of the base. At the next time step, the two clockwise rotating rollers are located farther upstream, with $\mathrm{C}$ following their motion. In the following time instant, it can be observed that $\mathrm{D}$ is deflected off the base in the radial direction towards the shear layer. Just shy of the body corner, the flow separates off the base and the structures impinge on the shear layer, introducing disturbances. Because the shear layer is convectively unstable, the perturbations experience amplification in the streamwise direction which most probably results in the generation of additional structures. The lack of azimuthal variation of $\mathrm{D}$ indicates that an axisymmetric stagnation point flow impinges on the base. This leads to a pressure peak at the axis, as shown in Sandberg \& Fasel (2006). At $t=t_{0}+3.2$, streamwise structures ('E') can be observed which closely resemble those found in figure 13 for the $1 / 8$-cylinder case at $R e_{D}=30000$. In the last time instance shown, even smaller axisymmetric rollers can be observed (' $F$ '), which in turn appear to generate additional streamwise structures within the recirculation region. Thus, it appears as if at least two separate mechanisms for the generation of longitudinal structures are present within the recirculation zone: (i) as discussed for the $R e_{D}=30000$ case, global modes lead to the generation of longitudinal structures within the recirculation region; (ii) braid-like structures appear to be generated in the presence of axisymmetric rollers and are, therefore, most probably a consequence of a centrifugal instability.

Figure 15 also reveals a significantly larger number of small-scale structures compared to the $R e_{D}=30000$ case. This was expected because of a broader range of unstable modes and increased growth rates for increasing Reynolds numbers. However, a qualitative difference was also observed at the highest Reynolds number case investigated. Streamwise structures appeared within the initial shear layer. The visualization of iso-surfaces of vorticity magnitude $\left|W_{i k}\right|=5$, shown in figure $16(a)$ confirms the presence of these streamwise vortices within the shear layer. The streamwise vortices are responsible for mushroom-shaped structures that can be observed in endviews of instantaneous local Mach number (figure 16b) at the streamwise location $z=3.5$.

These structures strongly resemble those visualized in experiments of a significantly higher Reynolds number flow at a comparable streamwise position (location C in Bourdon \& Dutton 1999). Over a longer time interval, approximately 12-14 mushroom-like structures were observed instantaneously, in good agreement with the typical number of 10-14 observed in the experiment. The streamwise structures undergo an amalgamation in the streamwise direction which intuitively seems inevitable in light of the lateral convergence of the shear layer. In the developing wake, at $z=6.5$ (corresponding to location $\mathrm{E}$ in Bourdon \& Dutton 1999), only four structures are visible for some instances, as in the experiments. This 'fourlobe' structure is a further indication that modes $k=2$ and $k=4$ are the dominant modes, both in the DNS at $R e_{D}=100000$ and experiments at $R e_{D}=3300000$, as will be explained in the following section. The perspective view of vorticity magnitude confirms that the streamwise structures do not persist throughout the recompression region. Instead, they seem to break up, forming a considerable number of hairpin vortices downstream of the recompression region. Because of the strong similarities between the DNS results and the experimental data, it is conjectured that the same instability mechanisms might be present. The resemblance of the results also suggests 

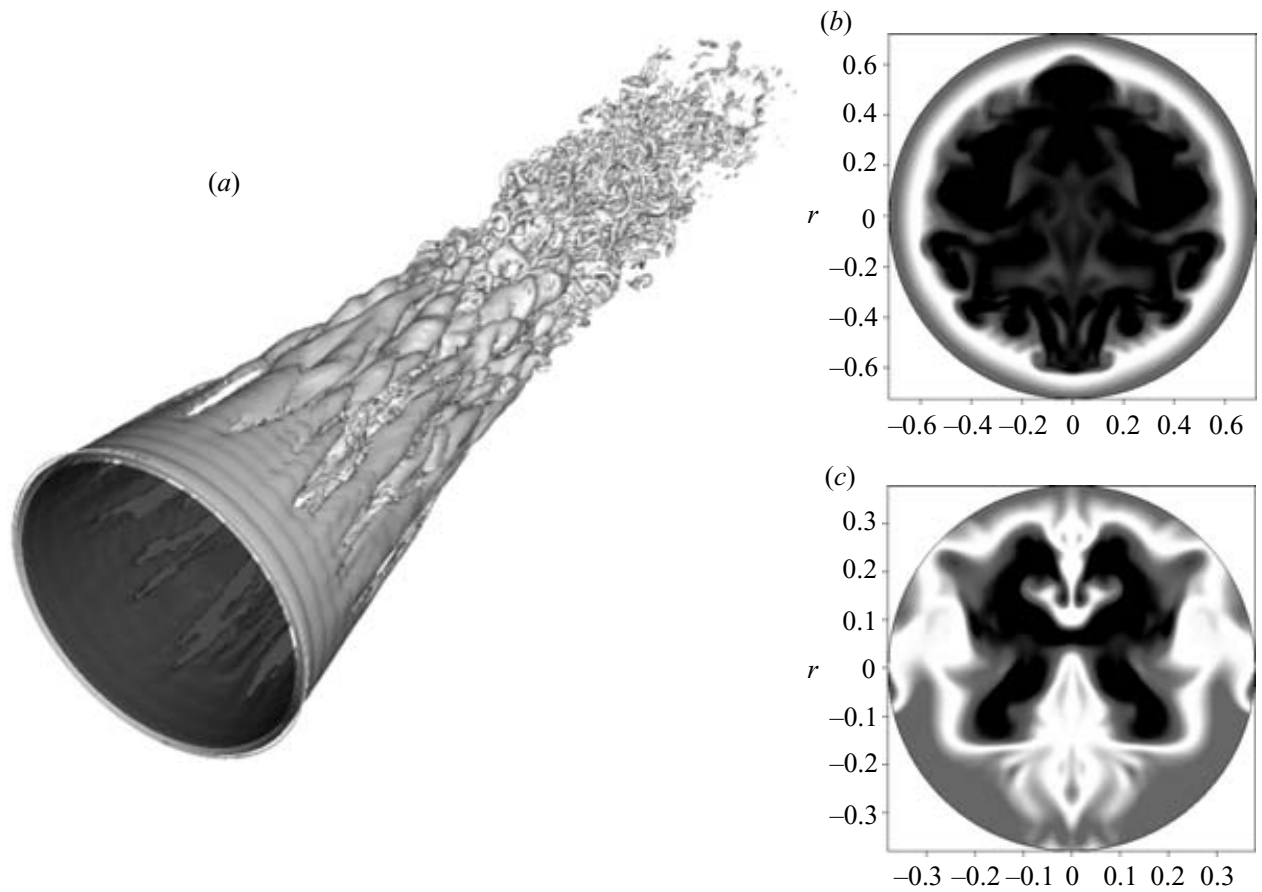

FIGURE 16. Instantaneous perspective view of iso-contours of vorticity magnitude $\left|W_{i k}\right|=5(a)$, and endviews of instantaneous contours of $M$ at $(b) z=3.5$ and $(c) z=6.5$ for half-cylinder case; $\operatorname{Re}_{D}=100000, M=2.46$.

that the absolute instability of the flow with respect to the axisymmetric mode $k=0$ that was observed for $R e_{D}>10000$ (see discussion in $\S 4.1 .2$ ) does not alter the wake significantly. It is presumed that the reason for this lies in the unsteady nature of the flow that leads to a continuous excitation of the zeroth mode which is convectively unstable at lower Reynolds numbers.

In addition, compressibility itself also might be responsible for the generation of vortical structures. The vorticity equation for compressible flows distinguishes itself from the incompressible formulation through an additional term, the baroclinic torque. The misalignment of the density gradients with the pressure gradients can lead to vorticity generation and destruction. At the base corner, for example, the rapid expansion produces strong density and pressure gradients. These two gradients are not aligned with each other and, therefore, the $r-z$, or axisymmetric, component of the baroclinic torque will be non-zero. As already stated above, axisymmetric disturbances are not significantly amplified in this region owing to the compressibility of the flow. However, once structures are present within the flow field, instantaneous density and pressure gradients will be present that move with the structures and produce all components of the baroclinic torque vector. Iso-contours of the magnitude of baroclinic torque $\left(1 / \rho^{2}|\nabla \rho \times \nabla p|=0.2\right)$ are shown in figure 17. For both Reynolds numbers shown, the main contribution of vorticity production or destruction can be found in the outer shear layer and the trailing wake, i.e. where the flow is supersonic and the density gradients are significantly larger than in low-speed regions. The axisymmetric distribution of the magnitude of baroclinic torque observed just downstream of the base can be attributed to the steady rapid expansion mentioned above, which results in a large contribution of the $r-z$-component. Further 

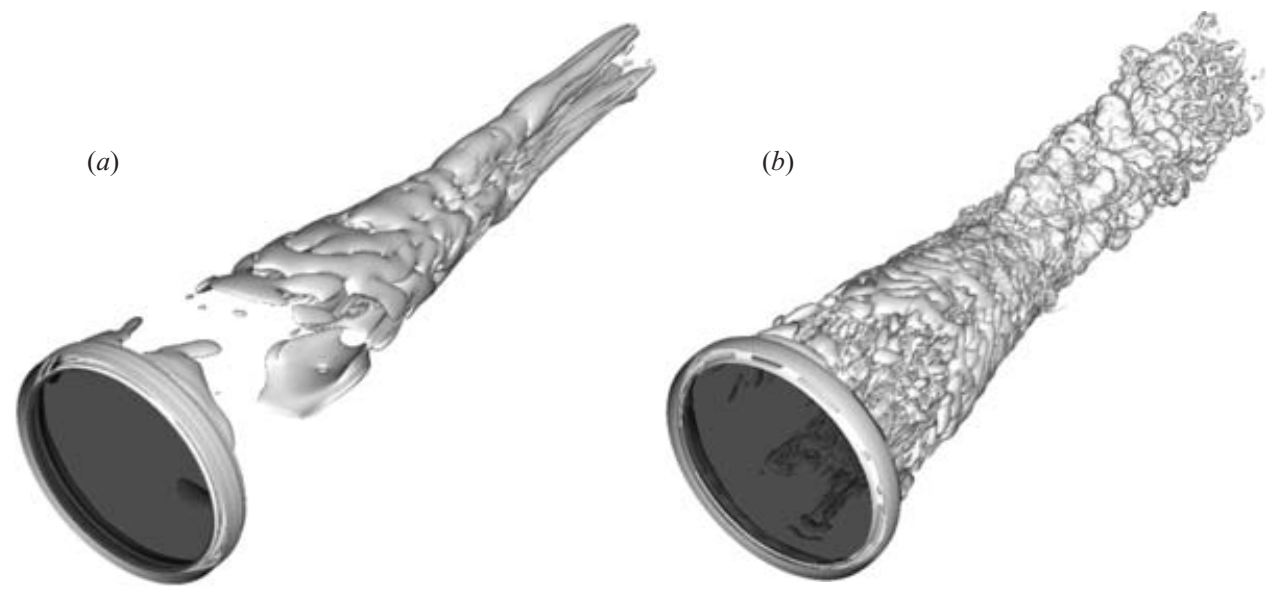

FIGURE 17. Instantaneous iso-contours of magnitude of baroclinic torque for half-cylinder case at $(a) R e_{D}=30000$ and $(b) R e_{D}=100000$; perspective view from inflow towards outflow; iso-level $0.2 ; M=2.46$.

downstream, the helical nature of the instantaneous structures can be observed with the degree of obliqueness decreasing in the downstream direction. This might be due to the diminishing convective Mach number, leading to a stronger axisymmetric organization of the structures. At $R e_{D}=100000$ (figure 17b), the iso-surface of the magnitude of baroclinic torque becomes considerably more complex, connected to the much larger number of structures. In the trailing wake, a distribution can be observed that suggests the presence of hairpin vortices. This led Terzi et al. (2006) to classify baroclinic torque as a suitable vortex identification criterion. It can also be observed that just downstream of separation, 'patches' with a regular azimuthal spacing are present. These might be connected to the generation of the longitudinal vortices visible in contours of vorticity magnitude. Upstream of the recompression region, it seems as if mainly the axisymmetric vorticity component is produced by the baroclinic torque mechanism, albeit experiencing a strong circumferential variation.

The visualization of coherent structures demonstrated that helical structures are present within the initial shear layer, confirming results from linear local analysis with the temporal code. Circumstantial evidence was presented, showing that longitudinal structures within the recirculation region are generated as a consequence of global modes reaching large amplitudes. Additionally, it was suggested that these structures might be generated by centrifugal instabilities associated with axisymmetric rollers. Endviews at $R e_{D}=100000$ were compared with data from experiments at a much higher Reynolds number and a remarkable resemblance was found. Finally, the baroclinic torque was identified as another important vorticity production mechanism.

\subsubsection{Mean flow}

To assess the impact of the large-scale structures, on the mean flow, averaged flow quantities must be evaluated. The DNS were conducted until running averages converged, which required approximately twelve flow-through times, i.e. $t \approx 12 \times$ $\left(z_{\text {outflow }}-z_{\text {inflow }}\right) / U_{\infty}$ for all cases. One of the figures of merit for judging which azimuthal Fourier modes are dominant and what effect they have on the mean flow is the visualization of endviews at different downstream locations. In order to give an impression of what kind of wake patterns (when looking at an endview) are 
(a)

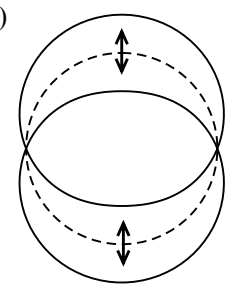

$k=1, A= \pm 0.4$

(e)

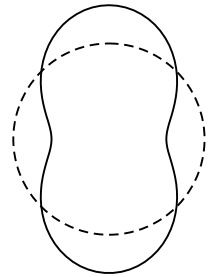

$k=2, A=0.4$ (b)

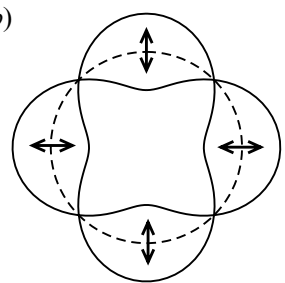

$k=2, A= \pm 0.4$

(f)

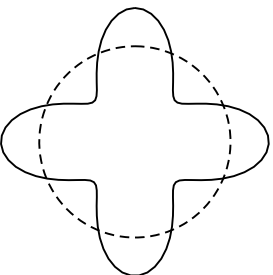

$k=4, A=0.4$ (c)

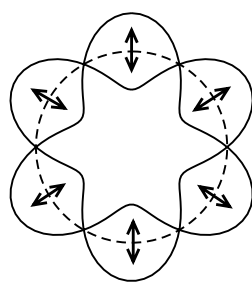

$k=3, A= \pm 0.4$

$(g)$

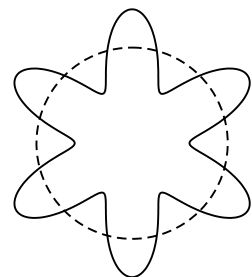

$k=6, A=0.4$ (d)

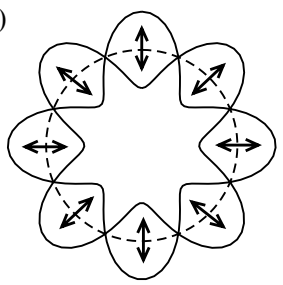

$k=4, A= \pm 0.4$

(h)

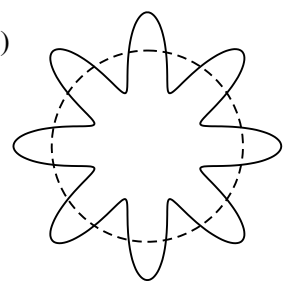

$k=8, A=0.4$

FIGURE 18. Schematic of wake patterns generated by various azimuthal modes.

generated by various azimuthal modes, several examples were computed analytically, superimposing individual azimuthal Fourier modes with the axisymmetric mode. For calculations employing symmetric Fourier transforms, scalar quantities, such as vorticity magnitude, are represented by a cosine series. The polar representation of the endview of the wake then becomes a function of the azimuthal mode number, $k$, the mean radial extent of the shear layer, $r_{0}$, chosen as unity here, and an amplitude, $A$, according to $r=r_{0}+A \cos (k \theta)$. Two fundamentally different solutions can be generated depending on how the amplitude $A$ is treated: the sign of $A$ can be kept constant, here the choice was a positive sign; or the sign of the constant can alternate, which would be the case if the respective mode exhibits an oscillatory behaviour in time. The resulting patterns are illustrated in figure 18 with the dashed line representing the undisturbed axisymmetric state. The results for an oscillating constant $A$ are shown in figure $18(a-d)$, and the solutions obtained when keeping the sign of $A$ constant are shown in figure 18(e-h). The same qualitative wake pattern can be produced with different modes. As shown in figures 18(a) and 18(e), a similar shape of wake can be generated through either a flapping of the wake in the lateral direction, caused by an oscillatory mode $k=1$, or the presence of a steady mode $k=2$. A 'fourlobe' structure is possible through either an oscillating mode $k=2$ or a dominant steady fourth mode as illustrated in figures $18(b)$ and $18(f)$. A 'six-lobe' pattern would be the consequence of either an oscillating dominant mode $k=3$ (figure 18c) or a steady mode $k=6$ (figure $18 g$ ), and in the case where the fourth mode (oscillatory, figure $18 d$ ) or $k=8$ (steady, figure 18h) exhibit the largest amplitudes, an 'eight-lobe' shape could occur. Naturally, any combination of the above patterns could also be generated if multiple azimuthal modes are significant in a particular case.

Figure 19 shows endviews of the time-averaged vorticity magnitude and the streamwise vorticity component of three azimuthal domain-sizes for the $\operatorname{Re}_{D}=30000$ case. Two characteristic streamwise locations are chosen. One region of interest is upstream of the recompression region, $z=2.5$, where the flow has not yet fully transitioned and considerable reverse flow occurs. The second region chosen is within the far wake, $z=9$, as the question arises whether the wake structure is affected by the strong adverse pressure gradient in the recompression region and by the highly 


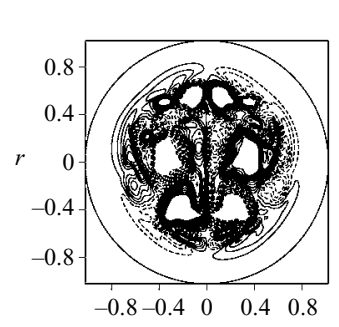

(a)
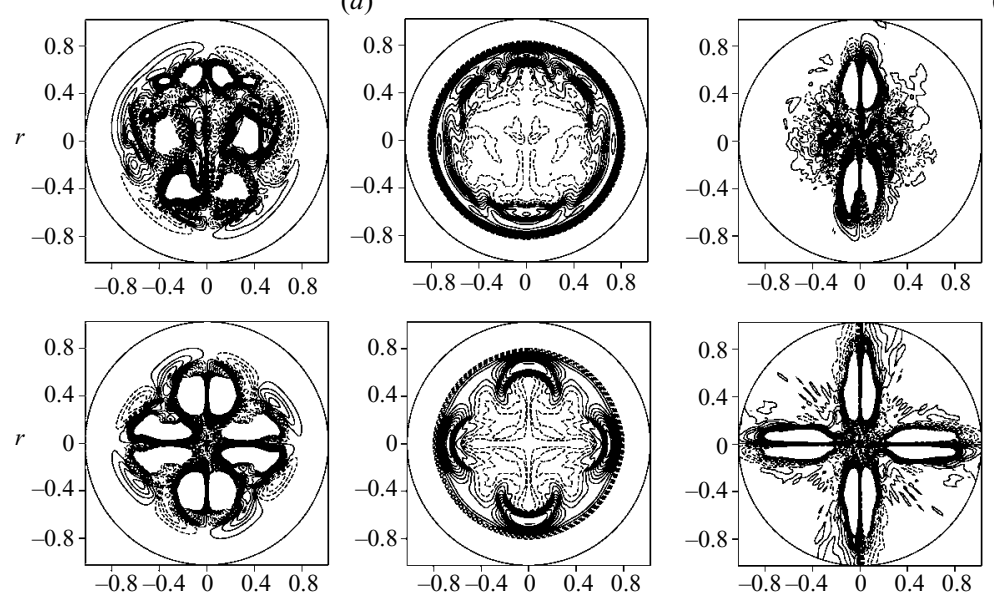

(b)
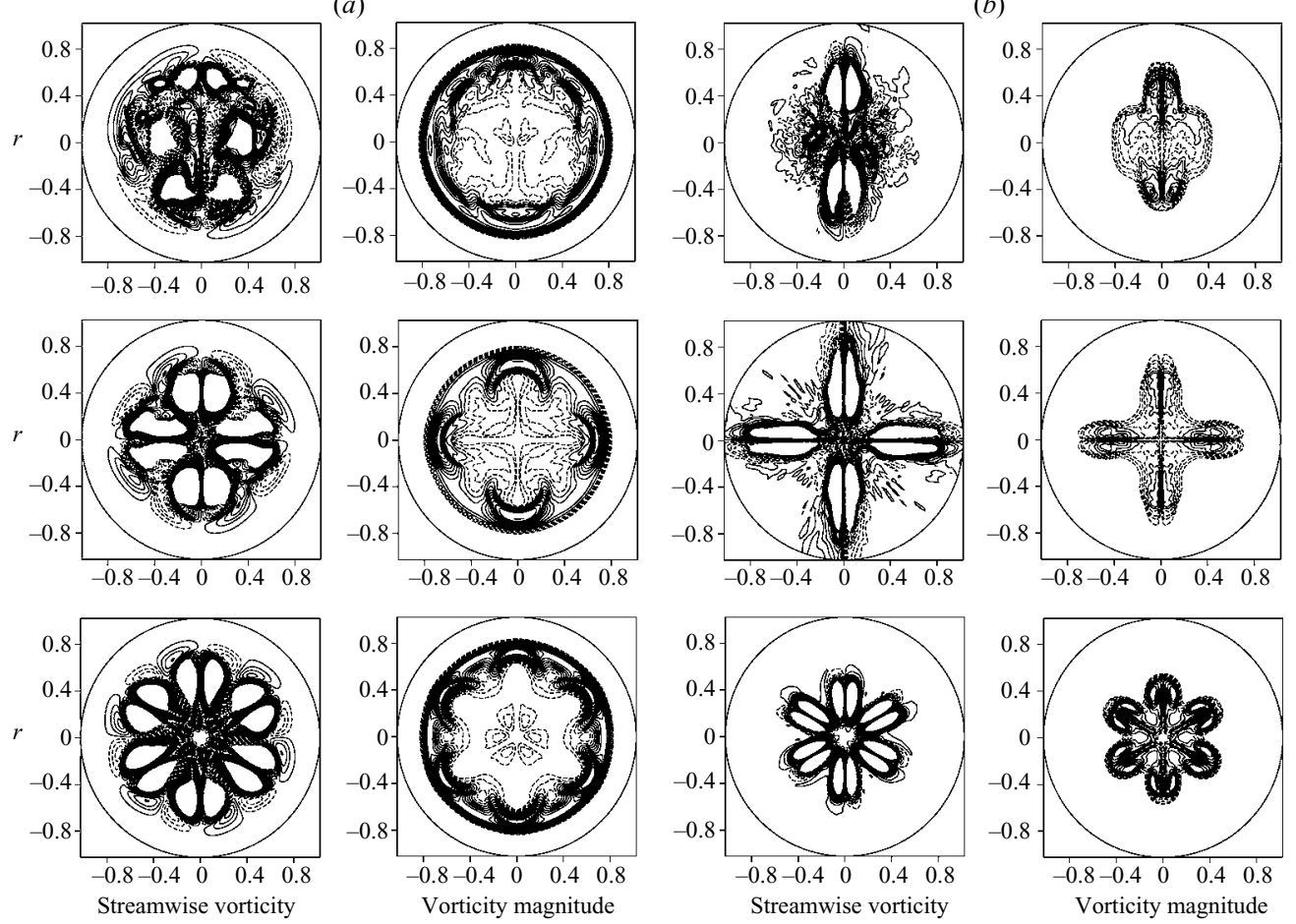

FIGURE 19. Endviews of time-averaged contours of vorticity magnitude and the streamwise vorticity component $(a)$ upstream $(z=2.5)$ and $(b)$ downstream $(z=9)$ of the recompression region for half-, quarter- and 1/6-cylinder (from top to bottom); for streamwise vorticity component, range $[-0.2 ; 0.2]$ with spacing 0.02 and negative values dashed; for vorticity magnitude, range $[0.8 ; 4]$ and $[0.8 ; 2.5]$ for $z=2.5$ and $z=9.0$, respectively, with spacing 0.2 and values smaller than 1.5 dashed; $R e_{D}=30000, M=2.46$.

unsteady flow behaviour. For the quarter- and 1/6-cylinder cases, the data from the calculations were mirrored and duplicated the respective number of times to show the results for the entire cylinder. This allows for a more convenient comparison between results for different domain sizes and provides a better impression of the resulting wake patterns. The shear layer (at $r \approx 0.7$ ) is most prominent in the visualization of vorticity magnitude, as this quantity includes the radial derivative of the streamwise velocity component. However, several distortions can be observed on the inside of the shear layer, which indicate that structures might be present in the mean flow. To assess whether these structures are of longitudinal nature, the focus is shifted to the visualization of the mean streamwise vorticity component, which does not include any component of the streamwise derivatives. As opposed to the vorticity magnitude, the streamwise vorticity component also contains information about the direction of rotation. The positive (solid lines) and negative (dashed lines) values denote clockwise and counterclockwise rotation, respectively.

Within the shear layer, negative values of the streamwise vorticity component are found in the mean flow for $0 \leqslant \theta \leqslant 2 \pi / 3$, pinched at $\theta \approx \pi / 3$. Positive values of the streamwise vorticity component are seen for $2 \pi / 3 \leqslant \theta \leqslant \pi$. In the interior of the recirculation region, the same behaviour can be observed, except for a reversed orientation and a more pronounced separation of the structures. The occurrence of only one sign change and the presence of distinctive features at $\theta \approx \pi / 3$ and $\theta \approx 2 \pi / 3$ 

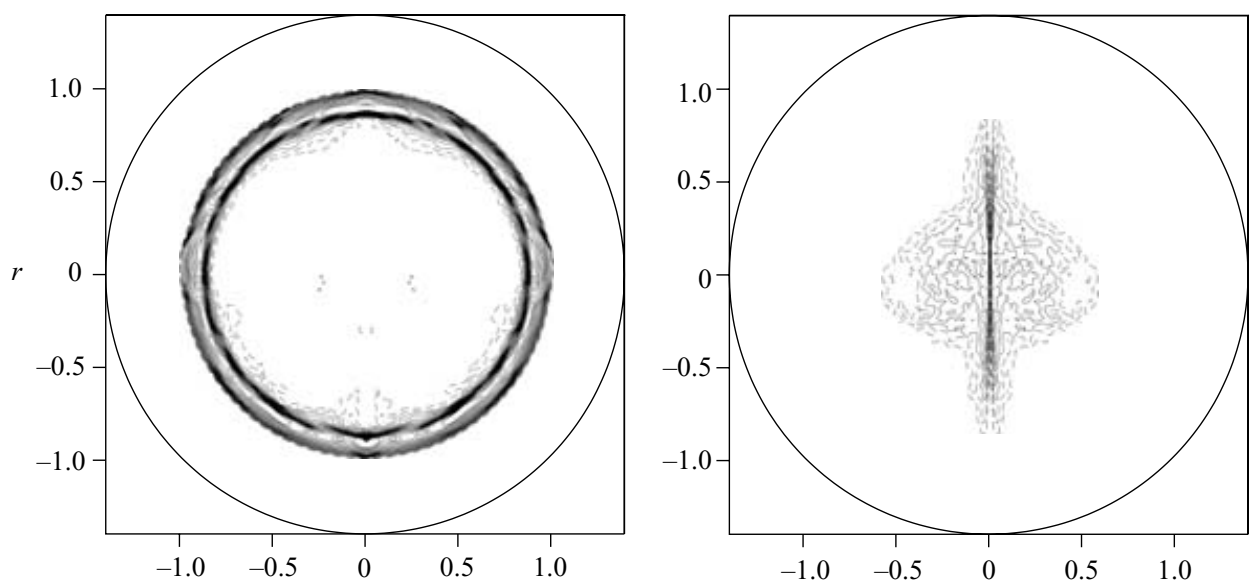

FIGURE 20. Endviews of time-averaged contours of vorticity magnitude $(a)$ upstream $(z=1.0)$ and $(b)$ downstream $(z=7.0)$ of the recompression region for half-cylinder; range $[1.0 ; 4.5]$ and $[1.0 ; 2.5]$ for $z=1.0$ and $z=7.0$, respectively, with spacing 0.2 and values smaller than 1.5 dashed; $R e_{D}=100000, M=2.46$.

implies that the mean flow is mainly the result of a superposition of modes $k=2$ and $k=3$.

For the other circumferential domain sizes, scrutinizing the visualizations of streamwise vorticity shows two pairs of counter-rotating structures within the integration domain. One pair is embedded in the shear layer, the other is present within the recirculation region, with a reversed orientation. When looking at contours of vorticity magnitude, it seems that for each pair of counter-rotating structures observed in contours of the streamwise vorticity component, one peak appears in the shear layer. This behaviour is caused by the entrainment and ejection of fluid from the recirculation region in the presence of longitudinal structures. The quarter-cylinder case therefore exhibits a 'four-lobe'-structure, the 1/6-cylinder calculation a 'six-lobe' structure as sketched in figure 18.

Downstream of recompression (figure 19b), the mean values of vorticity magnitude are reduced, mainly because the radial gradient of the streamwise velocity component is significantly smaller in the trailing wake than in the shear layer. The maximum values of the streamwise vorticity component remain roughly unchanged. This indicates that in the mean, the longitudinal structures persist throughout the strong pressure gradient in the recompression region. This supports the assumption that longitudinal structures are generated within the recirculation region and eventually travel downstream. In the half-cylinder case, a 'four-lobe' structure emerges, with the largest extension at $\theta=0$ and $\theta=\pi$. This can be attributed to a 'flapping' motion of the wake, which, because of the imposed symmetry, is restricted to the $\theta=0$ and $\theta=\pi$ plane. If full Fourier transforms were employed, the flapping movement could continuously select different planes and the average could therefore be more circular. This explanation is substantiated by the endview of the quarter-cylinder case, where mode $k=1$ is excluded and the 'four-lobe' structure is more prominent. The results from the 1/6-cylinder case also suggest that streamwise structures that form within the recirculation region persist into the developing wake, here causing a pronounced 'six-lobe' structure.

Endviews of time-averaged vorticity magnitude for simulations at $R e_{D}=100000$ are shown for $z=1$ and $z=7$ in figure 20. In contrast to the lower-Reynolds-number case, 
the visualization of contours of vorticity magnitude at $z=1$ reveals a strong azimuthal variation within the shear layer. This suggests that the streamwise structures reside in the shear layer over long periods of time. At the streamwise location $z=7$, as for the lower-Reynolds-number case a pronounced 'four-lobe' structure is visible. This implies that the 'four-lobed' wake pattern found in instantaneous endviews occurs not only on rare occasions, but is a frequently repeated process. For the smaller domain sizes, the results correspond to data that can be deduced from radial mode shapes (see discussion below). Thus, as the dominant modes of the respective case are the first subharmonic of the fundamental wavelength, a ' $k$-lobe' structure is visible, with $k$ being the number of the dominant mode. The results resemble those presented above for the $R e_{D}=30000$ case and are, therefore, not shown here.

From the observations made so far, no definite statement can be made about which modes cause the patterns seen. In order to determine which mode is responsible for the resulting wake pattern, additional data must be considered. Time-averaged radial amplitude distributions of several azimuthal Fourier modes for the streamwise velocity component are shown in figure 21 . In contrast to the radial distributions obtained from linear calculations, the amplitudes of the individual azimuthal modes obtained from DNS are relevant as they determine the degree of interaction between separate modes. Therefore, none of the modes is rescaled in the graphs to allow for a direct evaluation of the significance of individual modes.

When comparing the results obtained from DNS of the half-cylinder calculation at $R e_{D}=30000$ and $z=2.5$ (figure $21 a$ top) with the result presented for the linear calculations in figure 7, the following differences can be found. The shape of the first mode of the streamwise velocity component is significantly changed versus the result obtained in the linear calculation, showing similarities to several higher modes (regarding the locations of maxima, inflection points and slopes). Also, the overall values of the first mode are of the same order as those of the other dominant modes, in spite of a significantly smaller amplification rate determined in the linear calculations. This alludes to the possibility that the first azimuthal mode might be governed by nonlinear interaction of higher modes. Plots of the temporal development of the Fourier modes (not shown here) hinted at modes $k=3$ and $k=4$ most probably producing large amplitudes of $k=1$. The second azimuthal mode displays strongly altered mode shapes in comparison to the linear results. In contrast, the radial profiles of the third and fourth modes correspond very well with the data obtained from the linear calculations. This suggests that at this streamwise location (within the recirculation region, upstream of recompression), the azimuthal modes that were found to exhibit linearly the largest amplification rates also dominate in the nonlinear case. Recalling that the flow field appeared to be a result of modes $k=2$ and $k=3$ (see endviews in figure 19), it can be argued that mode $k=3$ is non-oscillatory in time (see discussion of figure 18).

Downstream of the recompression region, however, the picture changes drastically. When reviewing figure 21( $b$, top), it can be concluded that the radial distributions of all modes deviate noticeably from the linear solution, suggesting that a strong nonlinear interaction between all modes occurs in, or downstream of, the recompression region. This notion is supported by the observation that most modes shown in the graphs, except $k=1$, exhibit similar mode shapes. The maximum amplitude of the first mode is exceeded by the higher azimuthal modes, in particular modes $k=2,4,6$. Mode $k=3$ is not among the most significant modes, in spite of the largest growth rate found in the linear case (within the recirculation region) and the highest amplitudes at $z=2.5$. The strongly decreased magnitude of the third mode seen in the amplitude 
(a)
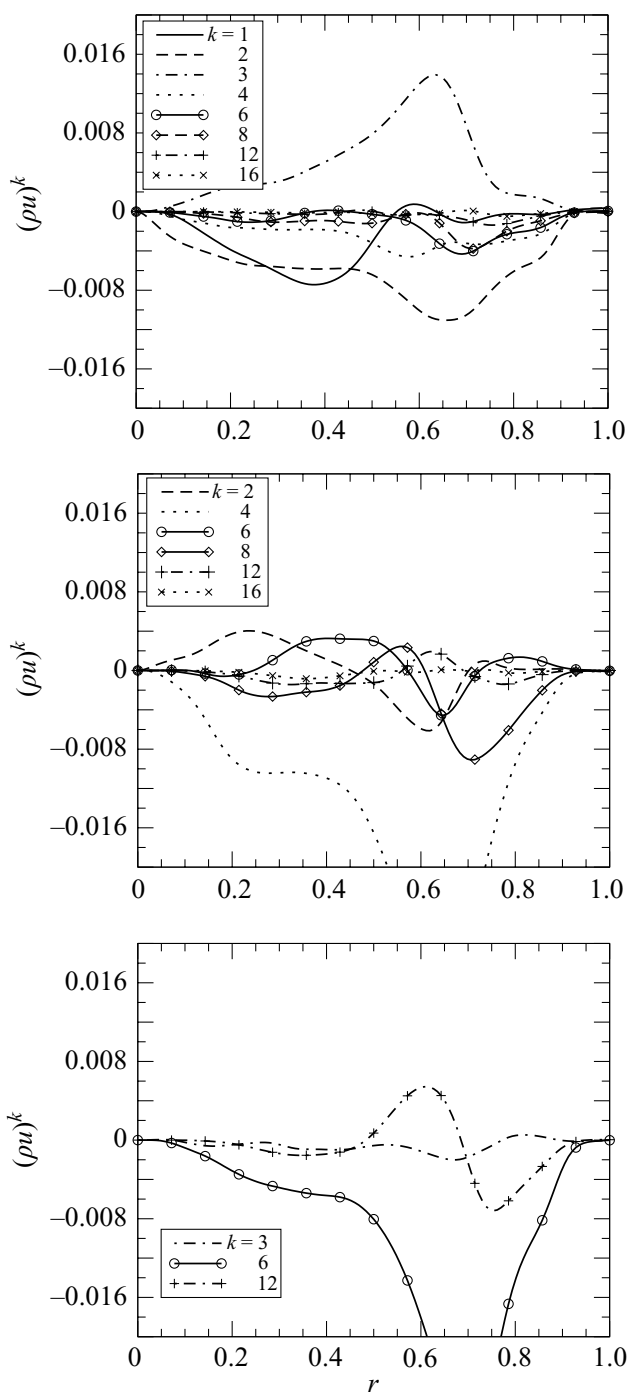

(b)
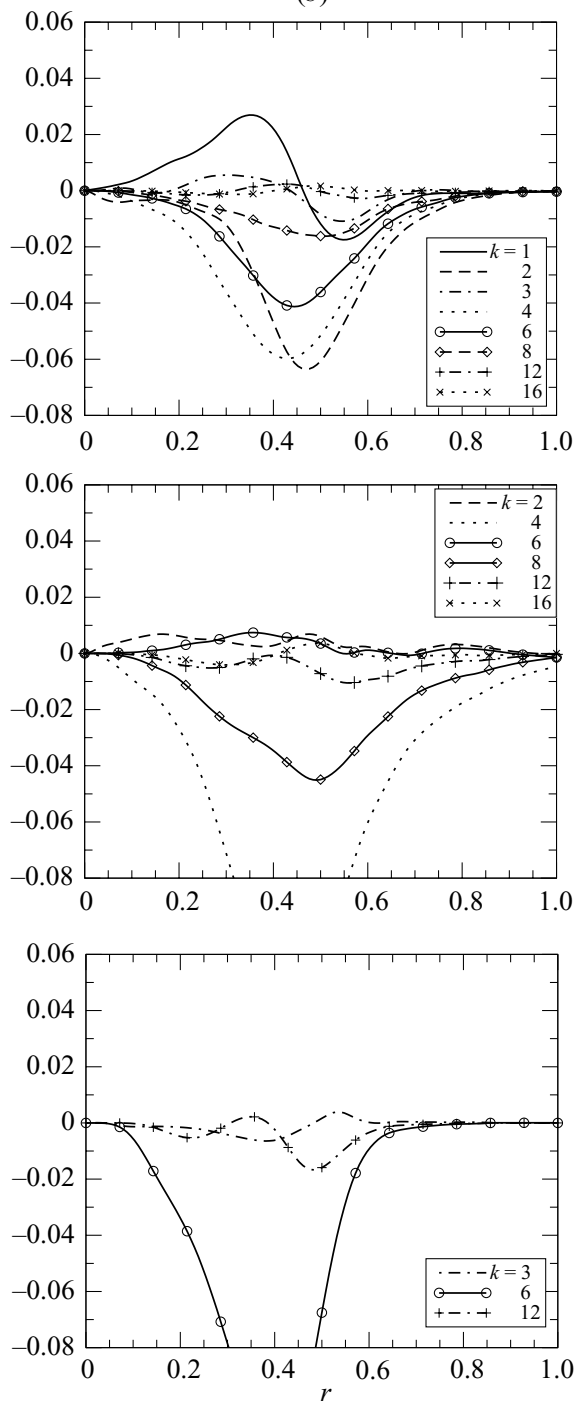

FIGURE 21. Time-averaged radial mode shapes of $(\rho u)^{k}$ obtained from DNS for half-, quarter- and 1/6-cylinder (from top to bottom); $(a) z=2.5,(b) z=9, \operatorname{Re}_{D}=30000, M=2.46$.

distribution at $z=9$ suggests that the wake structure appears to be governed by even modes. In fact, the 'four-lobe' structure observed in the endviews for this case implies that it is caused by an oscillatory mode $k=2$ and a steady mode $k=4$.

Now we will discuss the mode shapes obtained from DNS of different circumferential domain sizes. At $z=2.5$, when excluding all odd modes (quarter-cylinder calculation), the mode shape of $k=2$ is considerably different from the half-cylinder case; in fact, it resembles the profile of mode $k=4$. The fact that the shape of $k=2$ is strongly altered in the absence of $k=1$ indicates that the two modes strongly interact with each other in the half-cylinder case. This was also concluded in Sandberg \& Fasel (2006) when discussing the temporal spectra. The change in the amplitude distribution in the second mode, for the case where the first mode is omitted, is even more pronounced at $z=9$, especially for the streamwise component. The amplitude distributions of modes 
$k=4,6,8,12$ appear to remain fairly unchanged from the half-cylinder case for both streamwise locations, with $k=4$ exhibiting the largest maximum amplitude. Presumably, this is because, in the absence of all odd modes, energy from $k=0$ is transferred to the even modes through nonlinear interactions. In addition, mode $k=4$ has the largest (linear) growth rate of the modes included in the quarter-cylinder calculations.

For the 1/6-cylinder calculations, the most dominant mode is $k=6$, in contrast to the half-cylinder case, where, in the recirculation region, the amplitude of $k=3$ was considerably larger. For all domain sizes where mode $k=6$ is included, the same radial amplitude distribution is found, implying that this wavenumber does not significantly interact with wavenumbers that are excluded for domain sizes with $\theta_{\max }<\pi$.

Overall, the most striking observation when comparing the results for the different domain sizes with each other is that it is always the first higher harmonic of the fundamental wavelength that is the most dominant one, i.e. in the quarter-cylinder case the most dominant mode is $k=4$, in the $1 / 6$-cylinder case mode $k=6$, etc. Furthermore, regardless of the wavenumber, the mode shape of the most dominant mode is always the same for all domain sizes. For all domain sizes with $\theta<\pi$, the magnitude of the dominant mode is significantly larger than those in the half-cylinder case. This can be attributed to the fact that the dominant mode obtains energy directly from the spatial mean and does not have to 'share' this energy with the excluded modes through nonlinear interaction.

The averaged radial profiles of several azimuthal Fourier modes for the streamwise conservative velocity component at $R e_{D}=100000$ are shown in figure 22. The amplitude distribution of the first azimuthal mode at $z=2.5$ is similar to that found in the linear calculations (see figure 7), suggesting that $k=1$ is generated autonomously. This is in contrast to lower-Reynolds-number cases, where the shape found by DNS was fundamentally different from that obtained in the linear calculations, indicating that the first mode might be a subharmonic of some of the higher modes. As opposed to the lower-Reynolds-number cases, all higher modes exhibit their maxima at $z \approx 0.65$. This also confirms the findings of the linear stability calculations: the local convective shear-layer mode becomes increasingly dominant for larger Reynolds numbers. Several mode shapes resemble each other and possess similar amplitudes, with the most significant modes being $k=2,4,6,8$. The large amplitudes of higher modes within the shear layer constitute further evidence for the presence of longitudinal structures in the shear layer that were observed in the endviews.

For the downstream location (figure $22 b$ top), several qualitative differences compared to the lower Reynolds numbers can be observed. First, all modes exhibit smaller amplitudes, in particular the low-wavenumber modes. It is suggested that an increased energy transfer to higher modes, caused by the considerably increased level of turbulence, is the reason for this observation. Secondly, the maximum amplitude of the first azimuthal mode is close to an order of magnitude smaller than the dominant modes, even though it was the most dominant mode at the upstream position. In addition, it can be observed that modes $k=2$ and $k=4$ display the largest amplitudes in the mean. This confirms that they might be the dominant modes, as was supposed when observing a 'four-lobe' wake-pattern in endviews both instantaneously (figure 16) and for the mean flow (figure 20). The amplitude distributions obtained for all other domain sizes at $R e_{D}=100000$ are omitted for brevity. However, they again illustrate that for all domain sizes with $\theta_{\max }<\pi$, the first higher harmonic of the fundamental wavelength is the most dominant mode with the shape being highly similar for all cases.

For all Reynolds numbers that were investigated, $k=2$ and $k=4$ appeared to be the most dominant modes in the developing wake, and for all cases, a 'four-lobe' 
(a)
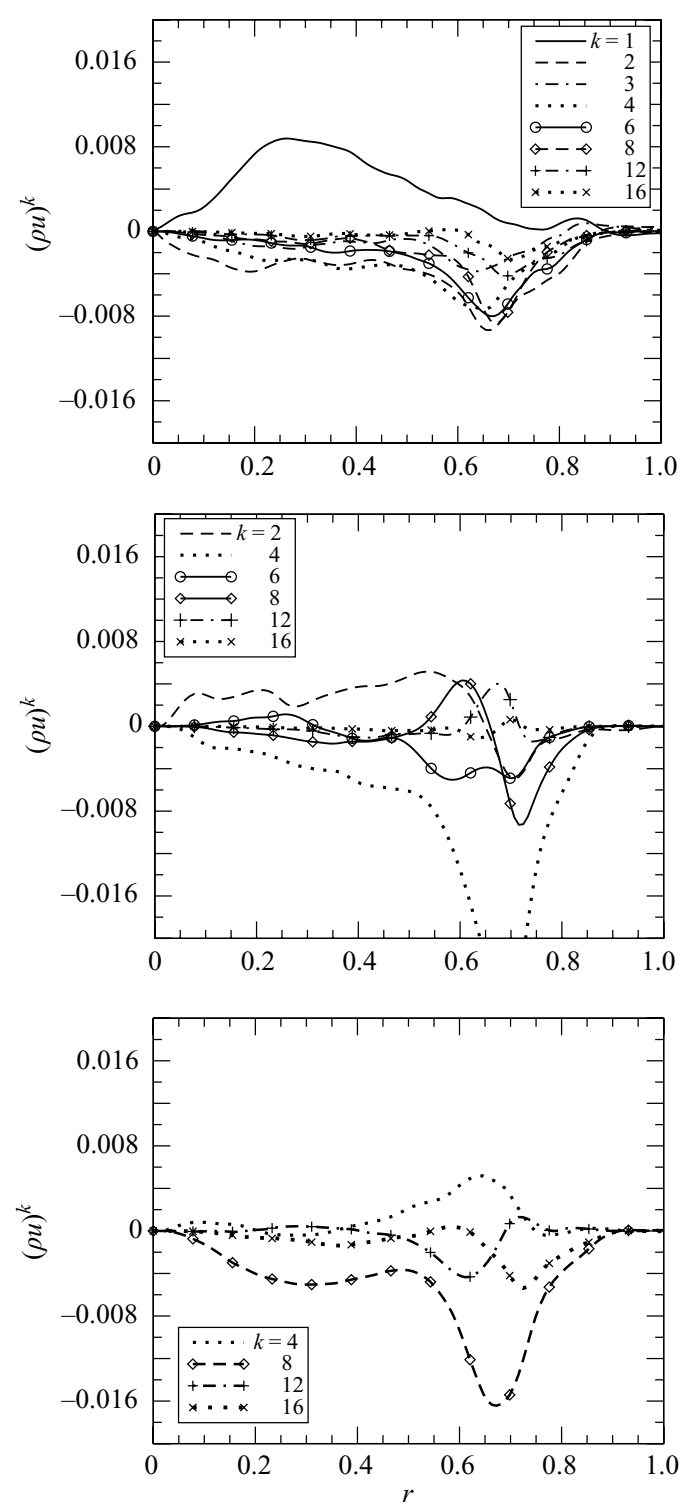

(b)
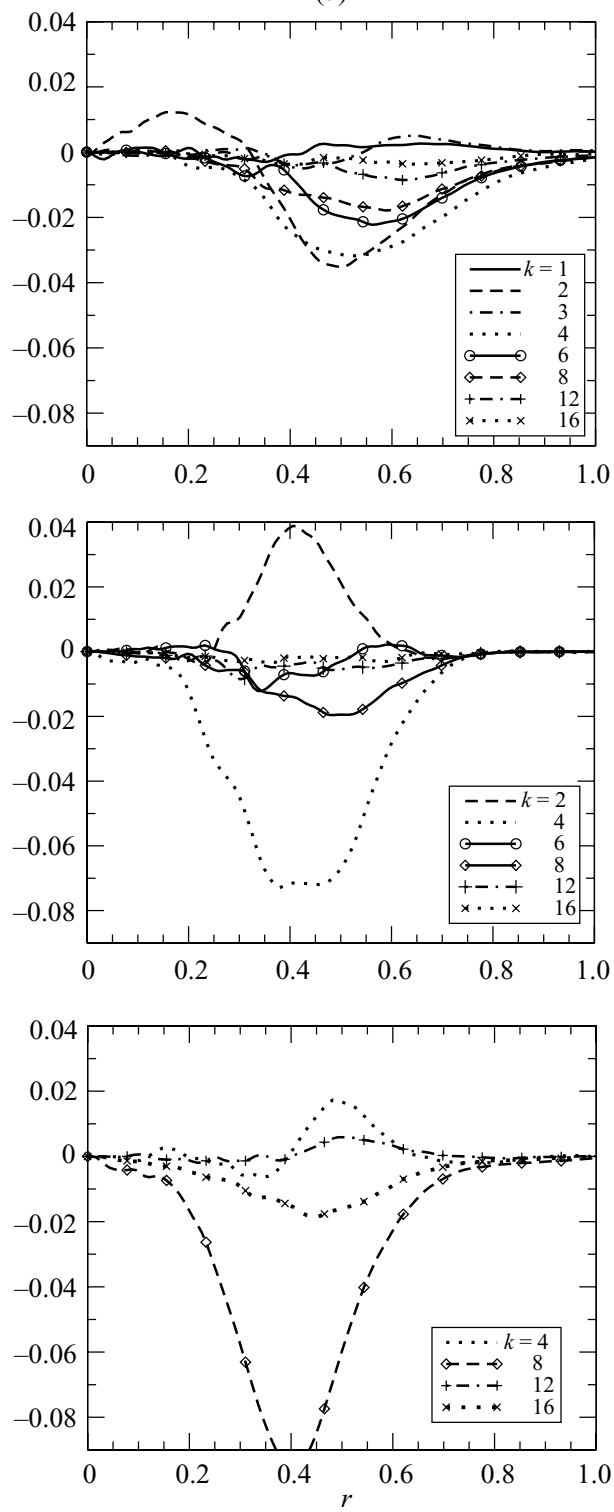

FiguRE 22. Time-averaged radial profiles of azimuthal Fourier modes of $(\rho u)$ obtained from DNS for half-, quarter- and 1/8-cylinder (from top to bottom); $(a) z=2.5,(b) z=7$, $R e_{D}=100000, M=2.46$.

wake structure was observed. Referring to the discussion of figure $18, k=2$ and $k=4$ are therefore oscillatory and steady modes, respectively. The occurrence of a steady mode $k=4$ contributing to the same wake pattern as an oscillatory mode $k=2$ can be explained as follows. Sandberg \& Fasel (2006) showed that the dominant azimuthal modes $k$ showed pronounced peaks in temporal spectra which can be denoted as temporal mode $n$. They further demonstrated that certain modes $(n, k)$ interact nonlinearly with themselves, producing either a response of the azimuthal mean with twice the frequency, $(n, k)+(n,-k)=(2 \times n, 0)$, or a higher azimuthal mode with twice 
the frequency $(n, k)+(n, k)=(2 \times n, 2 \times k)$. However, there is an additional scenario: a dominant mode interacting with itself also can produce $(n, k)-(n,-k)=(0,2 \times k)$. Thus, an oscillatory mode $k$ will most probably produce a time-steady mode $2 \times k$. These results suggest that this mechanism is quite strong, as evidenced by the large amplitudes found for the steady modes $2 \times k$. Furthermore, the fact that the first higher harmonic of the fundamental wavelength is the most dominant mode in all cases constitutes further evidence that this mechanism is highly effective.

\section{Conclusion}

Transitional axisymmetric wakes were investigated numerically using DNS, TDNS and LNS calculations. Particular emphasis was placed on identifying hydrodynamic instability mechanisms, and relating these to coherent structures that were identified with various visualization techniques. The premise for this approach was the assumption that flow instabilities lead to the formation of coherent structures.

Linear stability simulations were conducted of axisymmetric wakes at $M=2.46$ with an approach boundary-layer thickness $\delta_{c}=0.1$. For larger Reynolds numbers, an initial pulse disturbance led to temporal amplification of higher azimuthal modes $k>0$. It was concluded that the flow is absolutely unstable with respect to modes $k>0$ for $R e_{D}>5000$, with $k=3$ possessing the highest (linear) growth rate for Reynolds numbers up to $R e_{D}=100000$. In addition, for $R e_{D}>100000$, the flow becomes absolutely unstable with respect to the axisymmetric mode, $k=0$. By comparing spatial with temporal results, circumstantial evidence was found suggesting the co-existence of absolutely unstable global modes within the recirculation region and convectively unstable shear-layer modes. Mode shapes obtained from spatial simulations illustrated that the global mode is dominant for low Reynolds numbers. For increasing Reynolds numbers, the shear-layer mode appears to overcome the damping effect of compressibility and gains in importance. Local stability calculations revealed that the shear-layer modes have non-zero streamwise wavenumbers, implying that they are of a helical nature.

DNSs were carried out for three Reynolds numbers and $M=2.46$. Special emphasis was placed on identifying coherent structures and the most important modes for each case and their effect on the mean flow. This was accomplished by conducting simulations of various circumferential domain sizes, deliberately eliminating azimuthal/ helical modes. Thus, the effect of large-scale structures associated with particular azimuthal/helical modes on the global flow behaviour could be evaluated.

The $Q$-criterion was employed to identify vortical structures within the flow. For all Reynolds numbers investigated, helical structures within the initial shear-layer were detected. All cases revealed a considerable number of longitudinal structures within the recirculation region. It is proposed that at least two instability mechanisms are responsible for the generation of these structures. First, it is suggested that global modes cause a noticeable azimuthal modulation of the flow within the recirculation region. Therefore, a chevron-like pattern is imposed onto the recirculating fluid, leading to the formation of streamwise structures. Secondly, simulations of small circumferential domain sizes enabled the observation of axisymmetric rollers. These rollers result in instantaneous streamlines with strong curvature, most probably leading to a centrifugal instability which is responsible for the formation of braid-like longitudinal structures. Furthermore, structures within the recirculation region are entrained by the shear layer. The vortices are strongly stretched and develop into hairpin vortices. These hairpin vortices lead to additional instabilities responsible for the generation of small-scale structures. Local stability calculations using TDNS 
suggest that the helical structures present in the shear layer and the longitudinal structures in the trailing wake are a consequence of local instabilities. The baroclinic torque was identified as another important vorticity production mechanism. The largest contribution of vorticity production or destruction through baroclinic torque was found in regions subject to high compressibility, i.e. the initial shear-layer and the trailing wake.

Considerable evidence was found that the first azimuthal mode receives a significant amount of its energy through the nonlinear interaction of higher modes, mainly between $k=3$ and $k=4$. Only at $R e_{D}=100000$ does the first azimuthal mode $k=1$ appear to be generated autonomously. Another qualitative difference that could be observed at the highest Reynolds number simulated was the development of streamwise structures within the initial shear layer. These structures reside in the shear layer over long time periods, evidenced by their presence in time-averaged quantities. They lead to the presence of mushroom-shaped structures in the shear layer, observed in endviews. The mushroom-like structures are similar in shape and number to those detected in high-Reynolds-number experiments. At a location farther downstream, a 'four-lobe' wake pattern was observed, both instantaneously and in time-averaged data, in good agreement with experimental results. This 'four-lobe' pattern was found for all Reynolds numbers investigated, suggesting that modes $k=2$ and $k=4$ are the most important modes in the trailing wake. In this case, mode $k=2$ is oscillatory in time and through nonlinear interaction with itself produces the time-steady mode $k=4$ according to $(n, k)-(n,-k)=(0,2 \times k)$.

The results obtained from DNS at $R e_{D}=100000$ were shown to be highly similar to the experiments at $R e_{D}=3300000$. This implies that the most important hydrodynamic instability mechanisms dominating the flow in the experiments can be studied with DNS at the Reynolds numbers discussed here.

Work presented here was supported by the Army Research Office (ARO) under grant number DAAD 19-02-1-0361 \#1. Dr Thomas Doligalski served as program manager. Computer time on the ERDC/HPC under Challenge project VI9ARONC11312C1D is gratefully acknowledged.

\section{Appendix A. Navier-Stokes equations}

$$
\begin{aligned}
& \frac{\partial \boldsymbol{U}}{\partial t}+\frac{\partial \boldsymbol{A}}{\partial z}+\frac{\partial \boldsymbol{B}}{\partial r}+\frac{1}{r} \frac{\partial \boldsymbol{C}}{\partial \theta}+\frac{1}{r} \boldsymbol{D}=0 \quad \text { with } \quad \boldsymbol{U}=\left(\begin{array}{c}
\rho \\
\rho u \\
\rho v \\
\rho w \\
\rho E_{R}
\end{array}\right) \\
& \boldsymbol{A}=\left(\begin{array}{c}
\rho u \\
\rho u^{2}+p-\tau_{z z} \\
\rho u v-\tau_{r z} \\
\rho u w-\tau_{\theta z} \\
\rho u H_{R}+q_{z}-u \tau_{z z}-v \tau_{r z}-w \tau_{\theta z}
\end{array}\right), \boldsymbol{B}=\left(\begin{array}{c}
\rho v \\
\rho u v-\tau_{r z} \\
\rho v^{2}+p-\tau_{r r} \\
\rho v w-\tau_{r \theta} \\
\rho v H_{R}+q_{r}-u \tau_{r z}-v \tau_{r r}-w \tau_{r \theta}
\end{array}\right), \\
& \boldsymbol{C}=\left(\begin{array}{c}
\rho w \\
\rho u w-\tau_{\theta z} \\
\rho v w-\tau_{r \theta} \\
\rho w^{2}+p \tau_{\theta \theta} \\
\rho w H_{R}+q_{\theta}-u \tau_{\theta z}-v \tau_{r \theta}-w \tau_{\theta \theta}
\end{array}\right), \quad \boldsymbol{D}=\left(\begin{array}{c}
\rho v \\
\rho u v-\tau_{r z} \\
\rho v^{2}-\rho w^{2}-\tau_{r r}+\tau_{\theta \theta} \\
2 \rho v w-2 \tau_{r \theta} \\
\rho v H_{R}+q_{r}-u \tau_{r z}-v \tau_{r r}-w \tau_{r \theta}
\end{array}\right) .
\end{aligned}
$$


The individual components of the viscous stress-tensor and the strain-rate tensor are, respectively,

$$
\begin{aligned}
\tau_{z z} & =\frac{2 \mu}{R e}\left[S_{z z}-\frac{1}{3}\left(S_{z z}+S_{r r}+S_{\theta \theta}\right)\right], \quad \tau_{r r}=\frac{2 \mu}{R e}\left[S_{r r}-\frac{1}{3}\left(S_{z z}+S_{r r}+S_{\theta \theta}\right)\right], \\
\tau_{\theta \theta} & =\frac{2 \mu}{R e}\left[S_{\theta \theta}-\frac{1}{3}\left(S_{z z}+S_{r r}+S_{\theta \theta}\right)\right], \quad \tau_{r z}=\frac{2 \mu}{R e} S_{r z}, \quad \tau_{\theta z}=\frac{2 \mu}{R e} S_{\theta z}, \quad \tau_{r \theta}=\frac{2 \mu}{R e} S_{r \theta}, \\
S_{z z}= & \frac{\partial u}{\partial z}, \quad S_{r r}=\frac{\partial v}{\partial r}, \quad S_{\theta \theta}=\frac{1}{r} \frac{\partial w}{\partial \theta}+\frac{v}{r}, \\
S_{r z}= & \frac{1}{2}\left(\frac{\partial u}{\partial r}+\frac{\partial v}{\partial z}\right), \quad S_{\theta z}=\frac{1}{2}\left(\frac{\partial w}{\partial z}+\frac{1}{r} \frac{\partial u}{\partial \theta}\right), \quad S_{r \theta}=\frac{1}{2}\left(\frac{1}{r} \frac{\partial v}{\partial \theta}+r \frac{\partial}{\partial r} \frac{w}{r}\right) .
\end{aligned}
$$

\section{Appendix B. Derivation of linearized Navier-Stokes equations}

The derivation of the linearized Navier-Stokes equations is demonstrated in detail for the continuity equation. For brevity, only the final result is given for the remaining equations. The continuity equation is

$$
\frac{\partial}{\partial t}(\rho)+\frac{\partial}{\partial z}(\rho u)+\frac{\partial}{\partial r}(\rho v)+\frac{1}{r} \frac{\partial}{\partial \theta}(\rho w)+\frac{1}{r}(\rho v)=0 .
$$

Decomposing all variables into a basic state $(\breve{\phi})$ and a disturbance variable $(\dot{\phi})$, as described in $\S 2.3$, yields

$$
\begin{aligned}
\frac{\partial}{\partial t}(\breve{\rho}+\dot{\rho})+\frac{\partial}{\partial z}[(\breve{\rho} & +\dot{\rho})(\breve{u}+\dot{u})]+\frac{\partial}{\partial r}[(\breve{\rho}+\dot{\rho})(\breve{v}+\dot{v})] \\
& +\frac{1}{r} \frac{\partial}{\partial \theta}[(\breve{\rho}+\dot{\rho})(\breve{w}+\dot{w})]+\frac{1}{r}[(\breve{\rho}+\dot{\rho})(\breve{v}+\dot{v})]=0 .
\end{aligned}
$$

Subtracting products of basic-state variables, as they satisfy the continuity equation for the basic state, setting $\breve{w}=0$ and dropping all products containing more than one disturbance variable results in

$$
\frac{\partial}{\partial t}(\dot{\rho})+\frac{\partial}{\partial z}(\dot{\rho} \breve{u}+\breve{\rho} \dot{u})+\frac{\partial}{\partial r}(\dot{\rho} \breve{v}+\breve{\rho} \dot{v})+\frac{1}{r} \frac{\partial}{\partial \theta}(\breve{\rho} \dot{w})+\frac{1}{r}\left(\rho^{\prime} \breve{v}+\breve{\rho} \dot{v}\right)=0 .
$$

The final form of the linearized axial momentum equation is

$$
\begin{aligned}
& \frac{\partial}{\partial t}(\dot{\rho} \breve{u}+\breve{\rho} \dot{u})+\frac{\partial}{\partial z}\left(\breve{\rho} \breve{u} \dot{u}+\breve{\rho} \dot{u} \breve{u}+\dot{\rho} \breve{u} \breve{u}+\dot{p}-\dot{\tau}_{z z}\right)+\frac{\partial}{\partial r}\left(\breve{\rho} \breve{u} \dot{v}+\breve{\rho} \breve{u} \breve{v}+\dot{\rho} \breve{u} \breve{v}-\dot{\tau}_{r z}\right) \\
& +\frac{1}{r} \frac{\partial}{\partial \theta}\left(\breve{\rho} \breve{u} \dot{w}-\dot{\tau}_{\theta z}\right)+\frac{1}{r}\left(\breve{\rho} \breve{u} \dot{v}+\breve{\rho} \breve{u} \breve{v}+\dot{\rho} \breve{u} \breve{v}-\dot{\tau}_{r z}\right)=0,
\end{aligned}
$$

where the viscous stresses were linearized as follows:

$$
\dot{\tau}_{i k}=\frac{2 \breve{\mu}}{R e}\left[\dot{S}_{i k}-\frac{1}{3} \dot{S}_{j j} \delta_{i k}\right]+\frac{2 \dot{\mu}}{R e}\left[\breve{S}_{i k}-\frac{1}{3} \breve{S}_{j j} \delta_{i k}\right] .
$$

Using only the leading-order term of a Taylor series approximation of Sutherland's law, the linearized disturbance viscosity becomes

$$
\dot{\mu}=\breve{\mu} \dot{T}\left[\frac{3}{2 \breve{T}}-\frac{1}{\breve{T}+\mathscr{R}_{S u}}\right] \quad \text { with } \quad \breve{\mu}=\breve{T}^{\frac{3}{2}} \frac{1+\mathscr{R}_{S u}}{\breve{T}+\mathscr{R}_{S u}}
$$


Following the procedure discussed above, the final form of the linearized radial momentum, azimuthal momentum and energy equations are

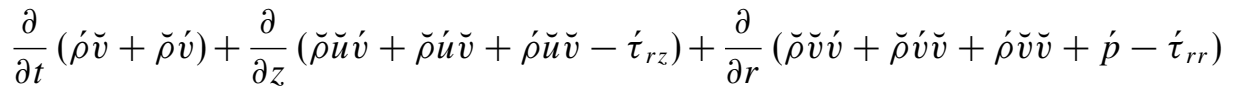

$$
\begin{aligned}
& +\frac{1}{r} \frac{\partial}{\partial \theta}\left(\breve{\rho} \breve{v} \dot{w}-\dot{\tau}_{r \theta}\right)+\frac{1}{r}\left(\breve{\rho} \breve{v} \dot{v}+\breve{\rho} v \underline{v}+\dot{\rho} \breve{v} \breve{v}-\dot{\tau}_{r r}+\dot{\tau}_{\theta \theta}\right)=0, \\
& \frac{\partial}{\partial t}(\breve{\rho} \dot{w})+\frac{\partial}{\partial z}\left(\breve{\rho} \breve{u} \dot{w}-\dot{\tau}_{\theta z}\right)+\frac{\partial}{\partial r}\left(\breve{\rho} \breve{v} \dot{w}-\dot{\tau}_{r \theta}\right) \\
& +\frac{1}{r} \frac{\partial}{\partial \theta}\left(\dot{p}-\dot{\tau}_{\theta \theta}\right)+\frac{1}{r}\left(2 \breve{\rho} \breve{v} \dot{w}-2 \dot{\tau}_{r \theta}\right)=0, \\
& \frac{\partial}{\partial t}(\breve{\rho} \dot{E}+\dot{\rho} \breve{E})+\frac{\partial}{\partial z}\left(\breve{\rho} \breve{u} \dot{H}+\breve{\rho} \breve{u} \breve{H}+\dot{\rho} \breve{u} \breve{H}+\dot{q}_{z}-\left(u \dot{\tau}_{z z}\right)-\left(v \dot{\tau}_{r z}\right)-\left(w \dot{\tau}_{\theta z}\right)\right) \\
& +\frac{\partial}{\partial r}\left(\breve{\rho} \breve{v} \dot{H}+\breve{\rho} \dot{v} \breve{H}+\dot{\rho} \breve{v} \breve{H}+\dot{q}_{r}-\left(u \dot{\tau}_{r z}\right)-\left(v \dot{\tau}_{r r}\right)-\left(w \dot{\tau}_{r \theta}\right)\right) \\
& +\frac{1}{r} \frac{\partial}{\partial \theta}\left(\breve{\rho} \dot{w} \breve{H}+\dot{q}_{\theta}-\left(u \dot{\tau}_{\theta z}\right)-\left(v \dot{\tau}_{r \theta}\right)-\left(w \dot{\tau}_{\theta \theta}\right)\right) \\
& +\frac{1}{r}\left(\breve{\rho} \breve{v} \dot{H}+\breve{\rho} \dot{v} \breve{H}+\dot{\rho} \breve{v} \breve{H}+\dot{q}_{r}-\left(u \dot{\tau}_{r z}\right)-\left(v \dot{\tau}_{r r}\right)-\left(w \dot{\tau}_{r \theta}\right)\right)=0 .
\end{aligned}
$$

The total energy of the basic state, the linearized total energy and the linearized heat-flux vector are

$$
\breve{E}=\frac{1}{\gamma E c} \breve{T}+\frac{1}{2}(\breve{u} \breve{u}+\breve{v} \breve{v}), \quad \dot{E}=\frac{1}{\gamma E c} \dot{T}+(\breve{u} \dot{u}+\breve{v} \dot{v}), \quad \dot{q}_{i}=-\frac{1}{\operatorname{PrEcRe}}\left(\breve{\kappa} \frac{\partial \dot{T}}{\partial x_{i}}+\dot{\kappa} \frac{\partial \breve{T}}{\partial x_{i}}\right),
$$

respectively. The total enthalpy of the basic state and the linearized total enthalpy, obtained by using a Taylor series approximation of the ratio of pressure and density are

$$
\breve{H}=\breve{E}+\frac{\breve{p}}{\breve{\rho}}, \quad \dot{H}=\dot{E}+\frac{\dot{p}}{\breve{\rho}}-\frac{\breve{p} \rho}{\breve{\rho}^{2}} .
$$

Finally, the linearized dissipation terms are

$$
\left(u_{i} \dot{\tau}_{i k}\right)=\breve{u}_{i} \dot{\tau}_{i k}+\dot{u}_{i} \breve{\tau}_{i k} \quad \text { with } \quad \breve{\tau}_{i k}=\frac{2 \breve{\mu}}{R e}\left[\breve{S}_{i k}-\frac{1}{3} \breve{S}_{j j} \delta_{i k}\right]
$$

In the linearized code, the variables

$$
\boldsymbol{U}=\left(\begin{array}{c}
U_{1} \\
U_{2} \\
U_{3} \\
U_{4} \\
U_{5}
\end{array}\right)=\left(\begin{array}{c}
\dot{\rho} \\
\dot{\rho} \breve{u}+\check{\rho} \dot{u} \\
\dot{\rho} \breve{v}+\breve{\rho} \dot{v} \\
\check{\rho} \dot{w} \\
\check{\rho} \dot{E}+\check{\rho} \breve{E}
\end{array}\right)
$$

are solved for. The primitive disturbance quantities are deduced from the conservative variables as follows

$\dot{u}=\frac{U_{2}-\dot{\rho} \breve{u}}{\breve{\rho}}, \quad \dot{v}=\frac{U_{3}-\grave{\rho} \breve{v}}{\breve{\rho}}, \quad \dot{w}=\frac{U_{4}}{\breve{\rho}}, \quad \dot{T}=\gamma E c\left[\frac{U_{5}-\dot{\rho} \breve{E}}{\breve{\rho}}-(\dot{u} \breve{u}+\dot{v} \breve{v})\right]$.

In order to close the system of equations, the disturbance pressure is then computed through the linearized equation of state for a perfect gas $\dot{p}=(\breve{\rho} \dot{T}+\dot{\rho} \breve{T}) / \gamma M^{2}$. 


\section{REFERENCES}

Aschenbach, E. 1974 Vortex shedding from spheres. J. Fluid Mech. 62, 209.

Bogdanoff, D. W. 1983 Compressibility effects in turbulent shear layers. AIAA J. 21, 926-927.

Bourdon, C. J. \& DutTon, J. C. 1999 Planar visualizations of large-scale turbulent structures in axisymmetric supersonic separated flows. Phys. Fluids 11, 201-213.

Cannon, S. \& Champagne, F. H. 1991 Large-scale structures in wakes behind axisymmetric bodies. In 8th Symposium on Turbulent Shear Flows, Munich, paper 6-5.

ChApmAn, D. R. 1951 An analysis of base pressure at supersonic velocities and comparison with experiments. NACA Rep. 1051. National Advisory Committee for Aeronautics.

Chung, Y. M. \& Tucker, P. G. 2003 Accuracy of high-order finite difference schemes on nonuniform grids. AIAA J. 41, 1609-1611.

Crow, S. C. \& Champagne, F. H. 1971 Orderly structure in jet turbulence. J. Fluid Mech. 48, $547-591$.

DaYman, B. 1963 Support interference effect on the supersonic wake. AIAA J. 8, 1921-1923.

Demetriades, A. $1968 a$ Turbulence front structure of an axisymmetric compressible wake. $J$. Fluid Mech. 34, 465-480.

Demetriades, A. $1968 b$ Turbulence measurements in an axisymmetric compressible wake. Phys. Fluids 11, 1841-1852.

DonalDSON, I. 1955 The effect of sting supports on the base pressure of a blunt-based body in a supersonic stream. Aeronaut. Q. 6, 221-229.

FASEL, H. F. 1990 Numerical simulation of instability and transition in boundary layer flows. In Laminar-Turbulent Transition, IUTAM Symposium, Toulouse 1989 (ed. D. Arnal \& R. Michel). Springer.

Fasel, H. F., von Terzi, D. A. \& Sandberg, R. D. 2006 A methodology for simulating compressible turbulent flows. J. Appl. Mech. 73, 405-412.

Gaster, M., Kit, E. \& Wygnanski, I. 1985 Large-scale structures in a forced turbulent mixing layer. J. Fluid Mech. 150, 23-39.

Hannemann, K. \& Oertel, H. 1989 Numerical simulation of the absolutely and convectively unstable wake. J. Fluid Mech. 199, 55-88.

Herbert, T. 1997 Parabolized stability equations. Annu. Rev. Fluid Mech. 29, 245-283.

Herrin, J. L. \& Dutton, J. C. 1994 Supersonic base flow experiments in the near wake of a cylindrical afterbody. AIAA J. 32, 77.

Herrin, J. L. \& Dutton, J. C. 1995 Effect of a rapid expansion on the development of compressible free shear layers. Phys. Fluids 7, 159-170.

Huerre, P. \& Monkewitz, P. A. 1985 Absolute and convective instabilities in free shear layers. J. Fluid Mech. 159, 151-168.

Huerre, P. \& Monkewitz, P. A. 1990 Local and global instabilities in spatially developing flows. Annu. Rev. Fluid Mech. 22, 473-537.

LAURIEN, E. \& KLEISER, L. 1989 Numerical simulation of boundary layer transition and transition control. J. Fluid Mech. 199, 403-440.

Lesieur, M. \& Metais, O. 1996 New trends in large-eddy simulations of turbulence. Annu. Rev. Fluid Mech. 28, 45-82.

Lewis, H. R. \& Bellan, P. M. 1990 Physical constraints on the coefficients of Fourier expansions in cylindrical coordinates. J. Math. Phys. 31, 2592-2596.

Marasli, B., Champagne, F. H. \& Wygnanski, I. 1989 Modal decomposition of velocity signals in a plane, turbulent wake. J. Fluid Mech. 198, 255.

Meitz, H. \& FaSel, H. F. 2000 A compact-difference scheme for the Navier-Stokes equations in vorticity-velocity formulation. J. Comput. Phys. 157, 371-403.

Monkewitz, P. 1988 A note on vortex shedding from axisymmetric bluff bodies. J. Fluid Mech. 192, $561-575$.

Oertel, H. 1979 Mach wave radiation of hot supersonic jets investigated by means of the shock tube and new optical techniques. Proc. 12th Intl. Symp. on Shock Tubes and Waves, Jerusalem (ed. A. Lifshitz \& J. Rom), pp. 266-275.

Oertel, H. 1990 Wakes behind blunt bodies. Annu. Rev. Fluid Mech. 22, 539-564.

Oertel, H. \& Delfs, J. 1995 Mathematische Analyse der Bereiche reibungsbehafteter Strömungen. Z. Angew. Math. Mech. 75, 491-505. 
Rayleigh, Lord 1916 On the dynamics of revolving fluids. Proc. R. Soc. Lond. A 93, 148-154, reproduced in Scientific Papers vol. 6, pp. 447-553. Cambridge University Press.

Rollstin, L. 1990 Measurement of inflight base-pressure on an artillery-fired projectile. J. Spacecraft Rockets 27, 5-6.

SANDBERG, R. D. 2004 Numerical investigation of transitional and turbulent supersonic axisymmetric wakes. $\mathrm{PhD}$ thesis, The University of Arizona.

SANDBerg, R. D. \& FASEl, H. 2004 Instability mechanisms in supersonic base flows. AIAA Paper 2004-0593.

SAndberG, R. D. \& Fasel, H. F. 2006 Direct numerical simulations of transitional supersonic base flows. AIAA J. 44, 848-858.

Schwarz, V., Bestek, H. \& Fasel, H. F. 1994 Numerical simulation of nonlinear waves in the wake of an axisymmetric bluff body. AIAA Paper 94-2285.

Terzi, D. A. von, Sandberg, R. D. \& Fasel, H. F. 2006 Identification of large coherent structures in compressible wake flows. J. Fluid Mech. (submitted).

Theofilis, V. 2003 Advances in global linear instability analysis of nonparallel and three-dimensional flows. Prog. Aerospace Sci. 39, 249-315.

White, F. M. 1991 Viscous Fluid Flow. McGraw-Hill.

Wygnanski, I., Champagne, F. H. \& Marasli, B. 1986 On large-scale coherent structures in two-dimensional, turbulent wakes. J. Fluid Mech. 168, 31. 